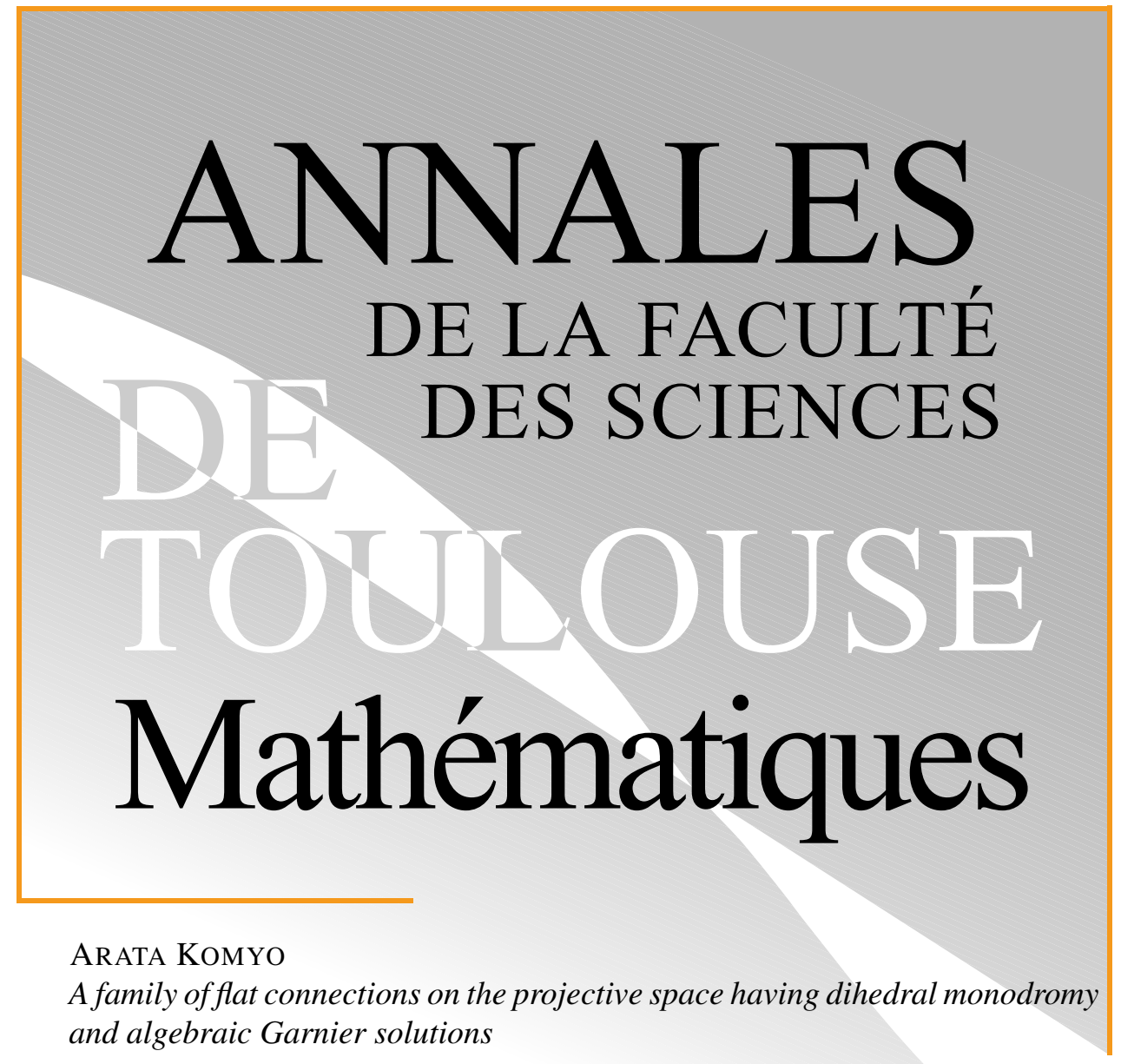

Tome XXX, no 3 (2021), p. 479-501.

https://doi.org/10.5802/afst.1682

(C) Université Paul Sabatier, Toulouse, 2021.

L'accès aux articles de la revue «Annales de la faculté des sciences de Toulouse Mathématiques » (http://afst.centre-mersenne.org/) implique l'accord avec les conditions générales d'utilisation (http://afst.centre-mersenne.org/legal/). Les articles sont publiés sous la license CC-BY 4.0.

(c)

Publication membre du centre

Mersenne pour l'édition scientifique ouverte MERSENNE http://www.centre-mersenne.org/ 


\title{
A family of flat connections on the projective space having dihedral monodromy and algebraic Garnier solutions ${ }^{(*)}$
}

\author{
Arata Komyo ${ }^{(1)}$
}

\begin{abstract}
A. Girand has constructed an explicit two-parameter family of flat connections over the complex projective plane $\mathbb{P}^{2}$. These connections have dihedral monodromy and their polar locus is a prescribed quintic composed of a conic and three tangent lines. In this paper, we give a generalization of this construction. That is, we construct an explicit $n$-parameter family of flat connections over the complex projective space $\mathbb{P}^{n}$. Moreover, we discuss the relation between these connections and the Garnier system.
\end{abstract}

RÉsumé. - A. Girand a construit une famille explicite de connexions à deux paramètres sur le plan projectif complexe $\mathbb{P}^{2}$. Ces connexions ont une monodromie diédrale et leur lieu polaire est une quintique prescrite composée d'une conique et de trois droites tangentes. Dans cet article, nous donnons une généralisation de cette construction. Autrement dit, nous construisons une famille de connexions plates explicite à $n$ paramètres sur l'espace projectif complexe $\mathbb{P}^{n}$. De plus, nous discutons de la relation entre ces connexions et le système Garnier.

\section{Introduction}

A meromorphic rank 2 connection $(E, \nabla)$ on a projective manifold $X$ is tha datum of a rank 2 vector bundle $E$ equipped with a $\mathbb{C}$-linear morphism $\nabla: E \rightarrow E \otimes \Omega_{X}^{1}(D)$ satisfying the Leibniz rule

$$
\nabla(f \cdot s)=f \cdot \nabla(s)+\mathrm{d} f \otimes s
$$

$\left.{ }^{*}\right)$ Reçu le 30 janvier 2019, accepté le 22 juillet 2019.

Keywords: algebraic function, Garnier system, isomonodromic deformation.

2020 Mathematics Subject Classification: 14H05, 14F35, 34M55.

(1) Center for Mathematical and Data Sciences, Kobe University, 1-1 Rokkodai-cho, Nada-ku, Kobe, 657-8501, Japan — akomyo@math.kobe-u.ac.jp

The author is supported by JSPS KAKENHI Grant Numbers 18J00245 and $19 \mathrm{~K} 14506$.

Article proposé par Bertrand Toën. 
for any section $s$ and function $f$. Here $D$ is the polar divisor of the connection $\nabla$. The connection $\nabla$ is flat when the curvature vanishes, that is $\nabla \cdot \nabla=0$. For a flat meromorphic rank 2 connection, we can define its monodromy representation. When $\operatorname{det}(E)=\mathcal{O}_{X}$ and the trace connection $\operatorname{tr}(\nabla)$ is the trivial connection on $\mathcal{O}_{X}$, we say that $(E, \nabla)$ is an $\mathfrak{s l}_{2}$-connection. A connection $(E, \nabla)$ is called regular if local $\nabla$-horizontal sections have moderate growth near the polar divisor $D$ (for details, see [4, Chapter II, Definition 4.2]).

In this paper, we introduce a family, parametrized by $\lambda \in \mathbb{C}^{n}$, of meromorphic $\mathfrak{s l}_{2}$-connections $\nabla_{\boldsymbol{\lambda}}=\mathrm{d}+A_{\boldsymbol{\lambda}}$ on the trivial bundle $\mathcal{O}_{\mathbb{P}^{n}} \oplus \mathcal{O}_{\mathbb{P}^{n}}$ over $\mathbb{P}^{n}$ with $n \geqslant 2$, with an explicit connection matrix $A_{\boldsymbol{\lambda}}$.

\subsection{The explicit expression of $\nabla_{\lambda}$}

The explicit connection matrix $A_{\boldsymbol{\lambda}}$ is described as follows. Let $[x: y$ : $\left.z_{1}: \ldots: z_{n-2}: t\right]$ be the homogeneous coordinates of $\mathbb{P}^{n}$. Set $f(x, y, t):=$ $x^{2}+y^{2}+t^{2}-2(x y+y t+t x)$. For $\boldsymbol{\lambda}=\left(\lambda_{0}, \ldots, \lambda_{n-1}\right) \in \mathbb{C}^{n}$, we define rational 1-forms on $\mathbb{P}^{n}$ as follows:

$$
\begin{aligned}
& \alpha_{0}(x, y):=-\frac{\left(2 \lambda_{0}+\lambda_{1}\right) \mathrm{d} x-\left(2 \lambda_{1}+\lambda_{0}\right) \mathrm{d} y}{2}-\frac{\lambda_{1}(y-1)}{2} \frac{\mathrm{d} x}{x}+\frac{\lambda_{0}(x-1)}{2} \frac{\mathrm{d} y}{y}, \\
& \alpha_{1}(x, y):=-\frac{1}{4} \frac{\mathrm{d} f(x, y, 1)}{f(x, y, 1)}, \quad \alpha_{2}(x, y):=-\frac{\alpha_{0}(x, y)}{f(x, y, 1)}
\end{aligned}
$$

and

$$
\begin{aligned}
& \alpha_{0}^{i}\left(x, y, z_{i}\right):=\lambda_{i+1}\left(\mathrm{~d} z_{i}-\frac{z_{i} \mathrm{~d}\left(f(x, y, 1)-z_{i}^{2}\right)}{2\left(f(x, y, 1)-z_{i}^{2}\right)}\right), \\
& \alpha_{2}^{i}\left(x, y, z_{i}\right):=-\frac{\alpha_{0}^{i}\left(x, y, z_{i}\right)}{f(x, y, 1)}
\end{aligned}
$$

which are described by the affine coordinates $\left[x: y: z_{1}: \ldots: z_{n-2}: 1\right]$. We define a connection matrix $A_{\boldsymbol{\lambda}}$ as

$$
A_{\boldsymbol{\lambda}}=\left(\begin{array}{cc}
\mathcal{A}_{11} & \mathcal{A}_{12} \\
-\mathcal{A}_{21} & -\mathcal{A}_{11}
\end{array}\right)+\sum_{i=1}^{n-2}\left(\begin{array}{cc}
\mathcal{A}_{11}^{i} & \mathcal{A}_{12}^{i} \\
-\mathcal{A}_{21}^{i} & -\mathcal{A}_{11}^{i}
\end{array}\right)
$$


where

$$
\begin{aligned}
& \mathcal{A}_{11}:=(x-1) \alpha_{2}(x, y)+\alpha_{1}(x, y)+\frac{1}{2} \frac{\mathrm{d} y}{y}, \\
& \mathcal{A}_{11}^{i}:=(x-1) \alpha_{2}^{i}\left(x, y, z_{i}\right), \\
& \mathcal{A}_{12}:=\frac{\mathrm{d} x+(x-1)^{2} \alpha_{2}(x, y)+2(x-1) \alpha_{1}(x, y)+\alpha_{0}(x, y)}{y}, \\
& \mathcal{A}_{12}^{i}:=\frac{(x-1)^{2} \alpha_{2}^{i}\left(x, y, z_{i}\right)+\alpha_{0}^{i}\left(x, y, z_{i}\right)}{y}, \\
& \mathcal{A}_{21}:=y \alpha_{2}(x, y), \\
& \mathcal{A}_{21}^{i}:=y \alpha_{2}^{i}\left(x, y, z_{i}\right),
\end{aligned}
$$

in the affine coordinates $\left[x: y: z_{1}: \ldots: z_{n-2}: 1\right]$.

\subsection{Main results}

Let $\mathcal{Q}_{0}$ and $\mathcal{Q}_{i}$ be the divisors on $\mathbb{P}^{n}$ defined by $\mathcal{Q}_{0}:=(f(x, y, t)=0)$ and $\mathcal{Q}_{i}:=\left(f(x, y, t)-z_{i}^{2}=0\right), i=1, \ldots, n-2$, respectively. Let $D_{n}$ be the divisor on $\mathbb{P}^{n}$ defined by

$$
D_{n}:=(x=0)+(y=0)+(t=0)+\mathcal{Q}_{0}+\mathcal{Q}_{1}+\cdots+\mathcal{Q}_{n-2} .
$$

From the explicit expression of $\nabla_{\boldsymbol{\lambda}}$, it follows that all $\nabla_{\boldsymbol{\lambda}}$ share the same polar divisor $D_{n}$. Note that the conic $\mathcal{Q}_{0}$ plays a special role: it is tangent to the conic $\mathcal{Q}_{i}$ inside the coordinate hyperplane $\left(z_{i}=0\right)$ for $i=1, \ldots, n-2$, and it is tangent to the three coordinate hyperplanes $(x=0),(y=0)$ and $(t=0)$.

TheOREm 1.1. - For each $\boldsymbol{\lambda}$, the connection $\nabla_{\boldsymbol{\lambda}}$ is flat and has at worst regular singularities.

We say that two connections $(E, \nabla)$ and $\left(E^{\prime}, \nabla^{\prime}\right)$ are birationally equivalent when there is a birational bundle transformation $\phi: E \rightarrow E^{\prime}$ that conjugates the two operators $\nabla$ and $\nabla^{\prime}$. We say that two connections $(E, \nabla)$ and $\left(E^{\prime}, \nabla^{\prime}\right)$ are projectively equivalent if the induced $\mathbb{P}^{1}$-bundles coincide $\mathbb{P}(E)=\mathbb{P}\left(E^{\prime}\right)$, and if moreover $\nabla$ and $\nabla^{\prime}$ induce the same projective connection $\mathbb{P}(\nabla)=\mathbb{P}\left(\nabla^{\prime}\right)$.

THEOREM 1.2. - Via a generically finite Galois morphism $f: \mathbb{P}^{n} \rightarrow \mathbb{P}^{n}$, for each $\boldsymbol{\lambda}$, the pull-back connection $f^{*} \nabla_{\boldsymbol{\lambda}}$ on the trivial bundle is projectively birationally equivalent to a split connection of the form

$$
\mathrm{d}+\left(\begin{array}{cc}
\omega & 0 \\
0 & -\omega
\end{array}\right)
$$

with $\omega$ a rational closed 1 -form on $X$. 
In this case, the generically finite Galois morphism is a genetically finite morphism of degree two. Loray, Pereira, and Touzet have proved the structure theorem of flat meromorphic $\mathfrak{s l}_{2}$-connections on projective manifolds in [9] (see also [1]). By Theorem 1.2, each $\nabla_{\boldsymbol{\lambda}}$ is the first type of the three possible types of flat meromorphic $\mathfrak{s l}_{2}$-connections over projective manifolds in the sense of Loray, Pereira, and Touzet [9, Theorem E].

Since the connection $\nabla_{\boldsymbol{\lambda}}$ is flat for each $\boldsymbol{\lambda}$, we can define its monodromy representation $\pi_{1}\left(\mathbb{P}^{n} \backslash D_{n}\right) \rightarrow \mathrm{SL}_{2}(\mathbb{C})$ of $\nabla_{\boldsymbol{\lambda}}$ for each $\boldsymbol{\lambda}$. Let $\boldsymbol{D}_{\infty}$ be the infinite dihedral group:

$$
\boldsymbol{D}_{\infty}:=\left\langle\left(\begin{array}{cc}
0 & \alpha \\
-\alpha^{-1} & 0
\end{array}\right),\left(\begin{array}{cc}
\beta & 0 \\
0 & \beta^{-1}
\end{array}\right) \mid \alpha, \beta \in \mathbb{C}^{*}\right\rangle \leqslant \mathrm{SL}_{2}(\mathbb{C}) .
$$

For the monodromy representation of $\nabla_{\boldsymbol{\lambda}}$, we have the following.

THEOREM 1.3. - For generic $\boldsymbol{\lambda}$, the monodromy representation of $\nabla_{\boldsymbol{\lambda}}$ is conjugated to an explicit representation

$$
\rho_{\boldsymbol{\lambda}}: \pi_{1}\left(\mathbb{P}^{n} \backslash D_{n}\right) \longrightarrow \mathrm{SL}_{2}(\mathbb{C}),
$$

which is virtually abelian, i.e. abelian after a finite cover of $\mathbb{P}^{n} \backslash D_{n}$, and takes values in the infinite dihedral group $\boldsymbol{D}_{\infty}$.

\subsection{Algebraic Garnier solution}

The $(2 n-2)$-variable Garnier system $\mathcal{G}_{2 n-2}$ is the completely integrable Hamiltonian system

$$
\mathcal{G}_{2 n-2}: \begin{cases}\frac{\partial \rho_{j}}{\partial t_{i}}=-\frac{\partial K_{i}}{\partial \nu_{j}} & i, j=1, \ldots, 2 n-2 \\ \frac{\partial \nu_{j}}{\partial t_{i}}=\frac{\partial K_{i}}{\partial \rho_{j}} & i, j=1, \ldots, 2 n-2,\end{cases}
$$

where

$K_{i}=-\frac{\Lambda\left(t_{i}\right)}{T^{\prime}\left(t_{i}\right)}\left[\sum_{k=1}^{2 n-2} \frac{T\left(\nu_{k}\right)}{\left(\nu_{k}-t_{i}\right) \Lambda^{\prime}\left(\nu_{k}\right)}\left\{\rho_{k}^{2}-\sum_{m=1}^{2 n} \frac{\theta_{m}-\delta_{i m}}{\nu_{k}-t_{m}} \rho_{k}+\frac{\kappa}{\nu_{k}\left(\nu_{k}-1\right)}\right\}\right]$

with $t_{2 n-1}=0, t_{2 n}=1, \kappa:=\frac{1}{4}\left\{\left(\sum_{m=1}^{2 n} \theta_{m}-1\right)^{2}-\left(\theta_{\infty}^{2}+1\right)\right\}, \Lambda(t):=$ $\prod_{k=1}^{2 n-2}\left(t-\nu_{k}\right)$ and $T(t):=\prod_{k=1}^{2 n}\left(t-t_{k}\right)$ (see [5], [6] and [11]). Here $\theta_{m}$ $(m=1, \ldots, 2 n, \infty)$ is the constant parameters defined by

$$
\begin{aligned}
\theta_{1} & =\frac{1}{2}, \quad \theta_{2}=\frac{1}{2}, \quad \theta_{2 i+1}=\lambda_{i+1}, \quad \theta_{2 i+2}=\lambda_{i+1}(i=1, \ldots, n-2) \\
\theta_{2 n-1} & =\lambda_{1}, \quad \theta_{2 n}=\lambda_{0}-1, \quad \theta_{\infty}=\lambda_{0}+\lambda_{1} .
\end{aligned}
$$


To give a solution of the Garnier system $\mathcal{G}_{2 n-2}$, we consider the Fuchsian system with $2 n+1$ regular singularities at $0,1, t_{1}, \ldots, t_{2 n-2}, \infty$ :

$$
\mathrm{d}+\widetilde{H}_{2 n-1} \frac{\mathrm{d} \widetilde{x}}{\widetilde{x}}+\widetilde{H}_{2 n} \frac{\mathrm{d} \widetilde{x}}{\widetilde{x}-1}+\sum_{i=1}^{2 n-2} \widetilde{H}_{i} \frac{\mathrm{d} \widetilde{x}}{\widetilde{x}-t_{i}}
$$

where $\widetilde{H}_{i}(i=1, \ldots, 2 n)$ are $2 \times 2$ matrices independent of $\widetilde{x}$ and $t_{i} \neq t_{j}$ $(i \neq j)$. We assume that $\widetilde{H}_{2 n+1}:=-\sum_{i=1}^{2 n} \widetilde{H}_{i}$ is a diagonal matrix and the eigenvalues of $\widetilde{H}_{i}(i=1, \ldots, 2 n+1)$ are as in Table 1.1.

Table 1.1. The eigenvalues of the residue matrices $(i=1, \ldots, n-2)$.

\begin{tabular}{c|ccccccc} 
Reside matrices & $\widetilde{H}_{1}$ & $\widetilde{H}_{2}$ & $\widetilde{H}_{2 i+1}$ & $\widetilde{H}_{2 i+2}$ & $\widetilde{H}_{2 n-1}$ & $\widetilde{H}_{2 n}$ & $\widetilde{H}_{2 n+1}$ \\
\hline Eigenvalues & $\pm \frac{1}{4}$ & $\pm \frac{1}{4}$ & $\pm \frac{\lambda_{i+1}}{2}$ & $\pm \frac{\lambda_{i+1}}{2}$ & $\pm \frac{\lambda_{1}}{2}$ & $\pm \frac{\lambda_{0}-1}{2}$ & $\pm \frac{\lambda_{0}+\lambda_{1}}{2}$
\end{tabular}

We fix generators $\gamma_{\tilde{x}}\left(\widetilde{x}=0,1, t_{1} \ldots, t_{2 n-2}, \infty\right)$ of the fundamental group $\pi_{1}\left(\mathbb{P}^{1} \backslash\left\{0,1, t_{1}, \ldots, t_{2 n-2}, \infty\right\}, *\right)$. Here the loop $\gamma_{\tilde{x}}$ on $\mathbb{P}^{1}$ is oriented counterclockwise, $\widetilde{x}$ lies inside, while the other singular points lie outside. Let $\rho_{\boldsymbol{\lambda}}^{\prime}: \pi_{1}\left(\mathbb{P}^{1} \backslash\left\{t_{1}, \ldots, t_{2 n}, \infty\right\}, *\right) \rightarrow \mathrm{SL}_{2}(\mathbb{C})$ be the representation of the fundamental group defined by Table 1.2. If we have the isomonodromic deformation of the Fuchsian system (1.1) whose preserved monodromy representation is conjugated to $\rho_{\boldsymbol{\lambda}}^{\prime}$, then we obtain a solution of the Garnier system $\mathcal{G}_{2 n-2}$ (see [10, Section 2]).

Table 1.2. The representation of the fundamental group; here $a_{j}=$ $\exp \left(-\pi \sqrt{-1} \lambda_{j}\right) j=0,1, \ldots, n-1$.

$$
\begin{aligned}
& \begin{array}{c|c|c|c}
\gamma_{0} & \gamma_{1} & \gamma_{t_{1}} & \gamma_{t_{2}} \\
\hline\left(\begin{array}{cc}
a_{1} & 0 \\
0 & a_{1}^{-1}
\end{array}\right) & \left(\begin{array}{cc}
-a_{0} & 0 \\
0 & -a_{0}^{-1}
\end{array}\right) & \left(\begin{array}{cc}
0 & 1 \\
-1 & 0
\end{array}\right) & \left(\begin{array}{cc}
0 & a_{0}^{2} \\
-a_{0}^{-2} & 0
\end{array}\right)
\end{array} \\
& \begin{array}{c|c|c}
\gamma_{t_{2 i+1}}(i=1, \ldots, n-2) & \gamma_{t_{2 i+2}}(i=1, \ldots, n-2) & \gamma_{\infty} \\
\hline\left(\begin{array}{cc}
a_{i+1} & 0 \\
0 & a_{i+1}^{-1}
\end{array}\right) & \left(\begin{array}{cc}
a_{i+1}^{-1} & 0 \\
0 & a_{i+1}
\end{array}\right) & \left(\begin{array}{cc}
a_{0} a_{1}^{-1} & 0 \\
0 & a_{0}^{-1} a_{1}
\end{array}\right)
\end{array}
\end{aligned}
$$

We say $\left(\rho_{j}\left(t_{1}, \ldots, t_{2 n-2}\right), \nu_{j}\left(t_{1}, \ldots, t_{2 n-2}\right)\right)_{j=1, \ldots, 2 n-2}$ is an algebraic solution of $\mathcal{G}_{2 n-2}$ if $\left(\rho_{j}\left(t_{1}, \ldots, t_{2 n-2}\right), \nu_{j}\left(t_{1}, \ldots, t_{2 n-2}\right)\right)_{j=1, \ldots, 2 n-2}$ satisfies the Garnier system $\mathcal{G}_{2 n-2}$ and the graph of the solution has Zariski closure of dimension $2 n-2$.

THEOREM 1.4. - Let $T$ be a certain Zariski open subset of $\mathbb{A}^{2 n-2}$ parametrizing generic lines in $\mathbb{P}^{n}$. From the natural morphism $\mathbb{P}^{1} \times T \rightarrow \mathbb{P}^{n}$, one obtains a relative connection $\left(\nabla_{\mathbb{P}^{1} \times T / T}\right)_{\boldsymbol{\lambda}}$ with $2 n+1$ simple poles by the pull-back of $\nabla_{\boldsymbol{\lambda}}$. 
(i) Up to an étale base change $\widetilde{T} \rightarrow T$, an isomorphism of the relative trivial bundle, and up to relative Möbius transformations in the base, we can consider the relative connection $\left(\nabla_{\mathbb{P}^{1} \times T / T}\right)_{\boldsymbol{\lambda}}$ as a family of the Fuchsian system (1.1) parametrized by $T$.

(ii) The family $\left(\nabla_{\mathbb{P}^{1} \times T / T}\right)_{\boldsymbol{\lambda}}$ is isomonodromic. The preserved monodromy representation of the fundamental group $\pi_{1}\left(\mathbb{P}^{1} \backslash\left\{0,1, t_{1}, \ldots\right.\right.$, $\left.\left.t_{2 n-2}, \infty\right\}, *\right)$ of this isomonodromic family is conjugated to the representation given by Table 1.2

(iii) Since $\operatorname{dim} \widetilde{T}=2 n-2$, the connection matrices of the isomonodromic family $\left(\nabla_{\mathbb{P}^{1} \times T / T}\right)_{\boldsymbol{\lambda}}$ defines an algebraic solution of the Garnier system $\mathcal{G}_{2 n-2}$.

In the case of $n=2$, the family of connections $\nabla_{\boldsymbol{\lambda}}$ have been established by Girand in [7]. Moreover Girand have discussed an explicit relation to certain algebraic solutions of the sixth Painlevé equation in [7]. Our argument is generalization of Girand's idea of explicit construction of $\nabla_{\boldsymbol{\lambda}}$, and of the proof of the main results, to the case $n \geqslant 2$.

The organization of this paper is as follows. In Section 2, we introduce a family, parametrized by $\boldsymbol{\lambda} \in \mathbb{C}^{n}$, of meromorphic $\mathfrak{s l}_{2}$-connections $\nabla_{\boldsymbol{\lambda}}=d+A_{\boldsymbol{\lambda}}$ on the trivial bundle $\mathcal{O}_{\mathbb{P}^{n}} \oplus \mathcal{O}_{\mathbb{P} n}$ over $\mathbb{P}^{n}$ with $n \geqslant 2$, with an explicit connection matrix $A_{\boldsymbol{\lambda}}$. In Section 2.3, we show Theorem 1.1 and Theorem 1.2. In Section 3, we compute the monodromy representation of $\nabla_{\boldsymbol{\lambda}}$ for generic $\boldsymbol{\lambda}$. In Section 3.3, we show Theorem 1.3. In Section 4, we consider the natural morphism $\mathbb{P}^{1} \times T \rightarrow \mathbb{P}^{n}$, where $T$ is a certain Zariski open subset of $\mathbb{A}^{2 n-2}$ parametrizing generic lines in $\mathbb{P}^{n}$. Let $\left(\nabla_{\mathbb{P}^{1} \times T / T}\right)_{\boldsymbol{\lambda}}$ be the relative connection with $2 n+1$ simple poles given by the pull-back

of $\nabla_{\boldsymbol{\lambda}}$. In Section 4.1, we introduce an étale base change $\widetilde{T} \rightarrow T$ to prove the assertion (i) of Theorem 1.4. In Section 4.2, after the étale base change $\widetilde{T} \rightarrow T$, we compute the residue matrix of $\left(\nabla_{\mathbb{P}^{1} \times \tilde{T} / \tilde{T}}\right)_{\boldsymbol{\lambda}}$ for each simple pole. In Section 4.3, we recall the relation between isomonodromic deformations and the Garnier system following [10]. In Section 4.4, we show Theorem 1.4.

\section{Construction of flat connections on projective spaces}

In this section, we introduce a family, parametrized by $\boldsymbol{\lambda} \in \mathbb{C}^{n}$, of meromorphic $\mathfrak{s l}_{2}$-connections $\nabla_{\boldsymbol{\lambda}}=\mathrm{d}+A_{\boldsymbol{\lambda}}$ on the trivial bundle $\mathcal{O}_{\mathbb{P}^{n}} \oplus \mathcal{O}_{\mathbb{P}}$ over $\mathbb{P}^{n}$ with $n \geqslant 2$, with the explicit connection matrix $A_{\boldsymbol{\lambda}}$ described in Section 1.1. For this introduction, we start from a family, parametrized by $\boldsymbol{\lambda} \in \mathbb{C}^{n}$, of flat meromorphic $\mathfrak{s l}_{2}$-connections $\left(\nabla_{0}\right)_{\boldsymbol{\lambda}}$ on the trivial bundle $\mathcal{O}_{\mathbb{C}^{n}} \oplus \mathcal{O}_{\mathbb{C}^{n}}$ over $\mathbb{C}^{n}$ whose connection matrix splits. Next, we consider a birational transformation of the projective connection $\mathbb{P}\left(\left(\nabla_{0}\right)_{\boldsymbol{\lambda}}\right)$. We define a generically finite 
Galois morphism $f: \mathbb{C}^{n} \rightarrow \mathbb{C}^{n}$. We show that this birational transformation descend to a projective connection over $\mathbb{C}^{n}$. We denote by $\mathbb{P}\left(\left(\nabla_{1}\right)_{\boldsymbol{\lambda}}\right)$ this projective connection. The connection corresponding to $\mathbb{P}\left(\left(\nabla_{1}\right)_{\boldsymbol{\lambda}}\right)$ does not split. If we extend the projective connection $\mathbb{P}\left(\left(\nabla_{1}\right)_{\boldsymbol{\lambda}}\right)$ over $\mathbb{C}^{n}$ to a projective connection over $\mathbb{P}^{n}$ naively, then the extended projective connection over $\mathbb{P}^{n}$ has poles of oder 2 along the divisor $\mathbb{P}^{n} \backslash \mathbb{C}^{n}$. Then we consider a birational transformation of $\mathbb{P}\left(\left(\nabla_{1}\right)_{\boldsymbol{\lambda}}\right)$. By this birational transformation, we obtain the meromorphic $\mathfrak{s l}_{2}$-connections $\nabla_{\boldsymbol{\lambda}}=\mathrm{d}+A_{\boldsymbol{\lambda}}$ with the explicit connection matrix $A_{\boldsymbol{\lambda}}$ described in Section 1.1. Finally Theorem 1.1 and Theorem 1.2 follow from this construction of $\nabla_{\boldsymbol{\lambda}}$.

\subsection{Flat connections $\left(\nabla_{0}\right)_{\lambda}$ defined by rational closed 1 -forms}

Let $\lambda_{0}, \ldots, \lambda_{n-1}$ be complex numbers. Set $Y:=\operatorname{Spec} \mathbb{C}\left[u_{0}, u_{1}, z_{1}, \ldots, z_{n-2}\right]$. Let $\omega_{0}$ and $\psi_{n}$ be the closed rational 1-forms on $Y$ defined by

$$
\begin{aligned}
& \omega_{0}:=\lambda_{0}\left(\frac{\mathrm{d} u_{0}}{u_{0}}-\frac{\mathrm{d} u_{1}}{u_{1}}\right)+\lambda_{1}\left(\frac{\mathrm{d} u_{0}}{u_{0}-1}-\frac{\mathrm{d} u_{1}}{u_{1}-1}\right) \\
& \psi_{n}:= \begin{cases}\sum_{i=1}^{n-2} \lambda_{i+1}\left(\frac{\mathrm{d}\left(u_{0}-u_{1}+z_{i}\right)}{u_{0}-u_{1}+z_{i}}-\frac{\mathrm{d}\left(u_{0}-u_{1}-z_{i}\right)}{u_{0}-u_{1}-z_{i}}\right) & n>2 \\
0 & n=2 .\end{cases}
\end{aligned}
$$

We have a family of flat connections

$$
\left(\nabla_{0}\right)_{\boldsymbol{\lambda}}:=\mathrm{d}+\frac{1}{2}\left(\begin{array}{cc}
\omega_{0}+\psi_{n} & 0 \\
0 & -\omega_{0}-\psi_{n}
\end{array}\right)
$$

on the trivial rank 2 vector bundle $E_{0} \rightarrow Y$. The family $\left(\nabla_{0}\right)_{\boldsymbol{\lambda}}$ is parametrized by $\boldsymbol{\lambda}=\left(\lambda_{0}, \ldots, \lambda_{n-1}\right)$. On the associated projective bundle $\mathbb{P}\left(E_{0}\right)$, we have the associated projective connection $\mathbb{P}\left(\left(\nabla_{0}\right)_{\boldsymbol{\lambda}}\right)=\mathrm{d} w_{0}+\left(\omega_{0}+\psi_{n}\right) w_{0}$, where $w_{0}$ is a projective coordinate on the fibers.

\subsection{Descent of the connection $\left(\nabla_{0}\right)_{\lambda}$}

We consider the birational transformation of the projective connection $\mathbb{P}\left(\left(\nabla_{0}\right)_{\boldsymbol{\lambda}}\right)$ defined by $\Phi: \mathbb{P}\left(E_{0}\right) \rightarrow \mathbb{P}\left(E_{0}\right)$;

$$
\left(u_{0}, u_{1}, z_{1}, \ldots, z_{n-2},\left[w_{0}^{0}: w_{0}^{1}\right]\right) \longmapsto\left(u_{0}, u_{1}, z_{1}, \ldots, z_{n-2},\left[\widetilde{w}_{0}^{0}: \widetilde{w}_{0}^{1}\right]\right),
$$

where

$$
\frac{\widetilde{w}_{0}^{1}}{\widetilde{w}_{0}^{0}}=\left(u_{0}-u_{1}\right) \frac{w_{0}^{1}+w_{0}^{0}}{w_{0}^{1}-w_{0}^{0}} .
$$


The rational function $(2.1)$ is an invariant of the involution $\iota: \mathbb{P}\left(E_{0}\right) \rightarrow$ $\mathbb{P}\left(E_{0}\right)$;

$$
\iota:\left(u_{0}, u_{1}, z_{1}, \ldots, z_{n-2},\left[w_{0}^{0}: w_{0}^{1}\right]\right) \longmapsto\left(u_{1}, u_{0}, z_{1}, \ldots, z_{n-2},\left[w_{0}^{1}: w_{0}^{0}\right]\right),
$$

that is $\left(\widetilde{w}_{0}^{1} / \widetilde{w}_{0}^{0}\right) \circ \iota=\widetilde{w}_{0}^{1} / \widetilde{w}_{0}^{0}$ as functions on $\mathbb{P}\left(E_{0}\right)$. Put $w_{0}=w_{0}^{1} / w_{0}^{0}$ and $\widetilde{w}_{0}=\widetilde{w}_{0}^{1} / \widetilde{w}_{0}^{0}$. We can check the following proposition by direct computation.

Proposition 2.1. - We define a map $f: Y \rightarrow \mathbb{P}^{n}$ by

$$
\left(u_{0}, u_{1}, z_{1}, \ldots, z_{n-2}\right) \longmapsto\left[s_{1}: s_{2}: z_{1}: \ldots: z_{n-2}: 1\right],
$$

where $s_{1}=u_{0}+u_{1}$ and $s_{2}=u_{0} u_{1}$. The birational transformation $\left(\Phi^{-1}\right)^{*} \mathbb{P}\left(\left(\nabla_{0}\right)_{\boldsymbol{\lambda}}\right)$ on $\mathbb{P}\left(E_{0}\right)$ descends to a projective connection on $f(Y) \times$ $\mathbb{P}^{1} \rightarrow f(Y):$

$$
\begin{aligned}
\left(\Phi^{-1}\right)^{*} \mathbb{P}\left(\left(\nabla_{0}\right)_{\boldsymbol{\lambda}}\right)= & \frac{\mathrm{d} \widetilde{w}_{0}}{\mathrm{~d} w_{0}}\left(\mathrm{~d} w_{0}+\left(\omega_{0}+\psi_{n}\right) w_{0}\right) \\
= & \mathrm{d} \widetilde{w}_{0}+\left(\alpha_{2}\left(s_{1}, s_{2}\right)+\sum_{i=1}^{n-2} \alpha_{2}^{i}\left(s_{1}, s_{2}, z_{i}\right)\right) \widetilde{w}_{0}^{2} \\
& +2 \alpha_{1}\left(s_{1}, s_{2}\right) \widetilde{w}_{0}+\left(\alpha_{0}\left(s_{1}, s_{2}\right)+\sum_{i=1}^{n-2} \alpha_{0}^{i}\left(s_{1}, s_{2}, z_{i}\right)\right),
\end{aligned}
$$

where

$$
\begin{aligned}
\alpha_{0}\left(s_{1}, s_{2}\right):= & \frac{2 \lambda_{0}\left(1-s_{1}+s_{2}\right)+\lambda_{1}\left(-s_{1}+2 s_{2}\right)}{2\left(1-s_{1}+s_{2}\right)} \mathrm{d} s_{1} \\
& -\frac{\lambda_{0} s_{1}\left(1-s_{1}+s_{2}\right)+\lambda_{1} s_{2}\left(s_{1}-2\right)}{2 s_{2}\left(1-s_{1}+s_{2}\right)} \mathrm{d} s_{2}, \\
\alpha_{0}^{i}\left(s_{1}, s_{2}, z_{i}\right):= & \lambda_{i+1}\left(\mathrm{~d} z_{i}-\frac{z_{i} \mathrm{~d}\left(s_{1}^{2}-4 s_{2}-z_{i}^{2}\right)}{2\left(s_{1}^{2}-4 s_{2}-z_{i}^{2}\right)}\right), \\
\alpha_{1}\left(s_{1}, s_{2}\right):=- & \frac{1}{4} \frac{\mathrm{d}\left(s_{1}^{2}-4 s_{2}\right)}{s_{1}^{2}-4 s_{2}} \\
\alpha_{2}\left(s_{1}, s_{2}\right):=- & \frac{\alpha_{0}\left(s_{1}, s_{2}\right)}{s_{1}^{2}-4 s_{2}}, \\
\alpha_{2}^{i}\left(s_{1}, s_{2}, z_{i}\right):= & -\frac{\alpha_{0}^{i}\left(s_{1}, s_{2}, z_{i}\right)}{s_{1}^{2}-4 s_{2}} .
\end{aligned}
$$

The corresponding connection $\left(\nabla_{1}\right)_{\boldsymbol{\lambda}}$ on $f(Y) \times \mathbb{C}^{2} \rightarrow f(Y)$ is

$$
\begin{aligned}
\left(\nabla_{1}\right)_{\boldsymbol{\lambda}}=\mathrm{d}+\left(\begin{array}{cc}
\alpha_{1}\left(s_{1}, s_{2}\right) & \alpha_{0}\left(s_{1}, s_{2}\right) \\
-\alpha_{2}\left(s_{1}, s_{2}\right) & -\alpha_{1}\left(s_{1}, s_{2}\right)
\end{array}\right) & \\
& +\sum_{i=1}^{n-1}\left(\begin{array}{cc}
0 & \alpha_{0}^{i}\left(s_{1}, s_{2}, z_{i}\right) \\
-\alpha_{2}^{i}\left(s_{1}, s_{2}, z_{i}\right) & 0
\end{array}\right) . \\
& -486-
\end{aligned}
$$


We consider a relation between this connection and the connection $\left(\nabla_{0}\right)_{\boldsymbol{\lambda}}$. Let $\nabla_{0}^{\prime}$ be the meromorphic connection on $Y \times \mathbb{C} \rightarrow Y$ defined by $\nabla_{0}^{\prime}:=$ $\mathrm{d}-\frac{1}{2} \frac{\mathrm{d}\left(u_{0}-u_{1}\right)}{u_{0}-u_{1}}$. We define a matrix $M_{1}\left(u_{0}, u_{1}\right)$ on $Y$ by

$$
M_{1}\left(u_{0}, u_{1}\right):=\left(\begin{array}{cc}
-1 & -u_{0}+u_{1} \\
-1 & u_{0}-u_{1}
\end{array}\right) .
$$

Let $\nabla_{0}^{\prime \prime}$ be the meromorphic connection on $Y \times \mathbb{C}^{2} \rightarrow Y$ defined by

$$
\begin{aligned}
\nabla_{0}^{\prime \prime}:=\mathrm{d}+M_{1}\left(u_{0}, u_{1}\right)^{-1} \mathrm{~d} M_{1}\left(u_{0}, u_{1}\right) & \\
& +M_{1}\left(u_{0}, u_{1}\right)^{-1} \frac{1}{2}\left(\begin{array}{cc}
\omega_{0}+\psi_{n} & 0 \\
0 & -\omega_{0}-\psi_{n}
\end{array}\right) M_{1}\left(u_{0}, u_{1}\right) .
\end{aligned}
$$

Then we have

$$
f^{*}\left(\nabla_{1}\right)_{\lambda}=\nabla_{0}^{\prime \prime} \otimes \nabla_{0}^{\prime}
$$

Moreover, we consider the map $\mathbb{P}^{n} \rightarrow \mathbb{P}^{n} ;\left[s_{1}: s_{2}: z_{1}: \ldots: z_{n-2}: t\right] \mapsto$ $\left[x: y: z_{1}: \ldots: z_{n-2}: t\right]$, where $x:=t-s_{1}+s_{2}$ and $y:=s_{2}$. Set

$$
f(x, y):=x^{2}+y^{2}+1-2(x y+x+y) .
$$

Then the rational 1-forms (2.3) are transformed into

$$
\begin{aligned}
\alpha_{0}(x, y)= & -\frac{\left(2 \lambda_{0}+\lambda_{1}\right) \mathrm{d} x-\left(2 \lambda_{1}+\lambda_{0}\right) \mathrm{d} y}{2} \\
& -\frac{\lambda_{1}(y-1)}{2} \frac{\mathrm{d} x}{x}+\frac{\lambda_{0}(x-1)}{2} \frac{\mathrm{d} y}{y}, \\
\alpha_{0}^{i}\left(x, y, z_{i}\right)= & \lambda_{i+1}\left(\mathrm{~d} z_{i}-\frac{z_{i} \mathrm{~d}\left(f(x, y)-z_{i}^{2}\right)}{2\left(f(x, y)-z_{i}^{2}\right)}\right), \\
\alpha_{1}(x, y)= & -\frac{1}{4} \frac{\mathrm{d} f(x, y)}{f(x, y)}, \\
\alpha_{2}(x, y)= & -\frac{\alpha_{0}(x, y)}{f(x, y)}, \\
\alpha_{2}^{i}\left(x, y, z_{i}\right)= & -\frac{\alpha_{0}^{i}\left(x, y, z_{i}\right)}{f(x, y)},
\end{aligned}
$$

which are described by the affine coordinates $\left[x: y: z_{1}: \ldots: z_{n-2}: 1\right]$.

\subsection{Birational transformations of the connection $\left(\nabla_{1}\right)_{\lambda}$}

From the connection $\left(\nabla_{1}\right)_{\boldsymbol{\lambda}}$ on $f(Y) \times \mathbb{C}^{2} \rightarrow f(Y)$, we construct a connection on the trivial bundle $\mathbb{P}^{n} \times \mathbb{C}^{2} \rightarrow \mathbb{P}^{n}$ whose pole divisor is $D_{n}$. If we extend the rational 1-forms (2.5) to rational 1-forms on $\mathbb{P}^{n}$, then $\alpha_{0}(x, y)$, $\alpha_{0}^{i}\left(x, y, z_{i}\right)$ and $\alpha_{1}(x, y)$ have poles of order 2, 2 and 1 along the divisor 
$(t=0)$, respectively. On the other hand, the rational 1-forms $\alpha_{2}(x, y)$ and $\alpha_{2}^{i}\left(x, y, z_{i}\right)$ have no pole along the divisor $(t=0)$. So we consider a birational transformation of the projective connection (2.2) as follows. The $\mathrm{d} y / y$ part of the projective connection $(2.2)$ is

$$
\begin{aligned}
\mathrm{d} \widetilde{w}_{0}- & \frac{\lambda_{0}\left(\widetilde{w}_{0}-x+1\right)\left(\widetilde{w}_{0}+x-1\right)}{x+1} \frac{\mathrm{d} y}{y} \\
& +[\text { terms whose pole divisors do not contain the divisor }(y=0)] .
\end{aligned}
$$

Then we consider the following birational map

$$
\begin{aligned}
\mathbb{P}^{n} \times \mathbb{P}^{1} & \longrightarrow \mathbb{P}^{n} \times \mathbb{P}^{1} \\
\left(\left[x: y: z_{1}: \ldots: z_{n-2}: 1\right],\left[1: \widetilde{w}_{0}\right]\right) & \longmapsto\left(\left[x: y: z_{1}: \ldots: z_{n-2}: 1\right],[1: w]\right),
\end{aligned}
$$

where $\widetilde{w}_{0}-x+1=w y$. By this birational transformation (2.6), the projective connection $(2.2)$ is transformed into

$$
\begin{aligned}
\mathrm{d} w & +\left(\mathcal{A}_{21}(x, y)+\sum_{i=1}^{n-2} \mathcal{A}_{21}^{i}\left(x, y, z_{i}\right)\right) w^{2} \\
& +2\left(\mathcal{A}_{11}^{i}(x, y)+\sum_{i=1}^{n-2} \mathcal{A}_{11}^{i}\left(x, y, z_{i}\right)\right) w+\mathcal{A}_{12}^{i}(x, y)+\sum_{i=1}^{n-2} \mathcal{A}_{12}^{i}\left(x, y, z_{i}\right),
\end{aligned}
$$

where

$$
\begin{aligned}
\mathcal{A}_{21}(x, y) & :=y \alpha_{2}(x, y), \\
\mathcal{A}_{21}^{i}\left(x, y, z_{i}\right) & :=y \alpha_{2}^{i}\left(x, y, z_{i}\right), \\
\mathcal{A}_{11}(x, y) & :=(x-1) \alpha_{2}(x, y)+\alpha_{1}(x, y)+\frac{1}{2} \frac{\mathrm{d} y}{y}, \\
\mathcal{A}_{11}^{i}\left(x, y, z_{i}\right) & :=(x-1) \alpha_{2}^{i}\left(x, y, z_{i}\right), \\
\mathcal{A}_{12}(x, y) & :=\frac{\mathrm{d} x+(x-1)^{2} \alpha_{2}(x, y)+2(x-1) \alpha_{1}(x, y)+\alpha_{0}(x, y)}{y}, \\
\mathcal{A}_{12}^{i}\left(x, y, z_{i}\right) & :=\frac{(x-1)^{2} \alpha_{2}^{i}\left(x, y, z_{i}\right)+\alpha_{0}^{i}\left(x, y, z_{i}\right)}{y} .
\end{aligned}
$$

The corresponding connection $\nabla_{\boldsymbol{\lambda}}$ on $\mathbb{P}^{n} \times \mathbb{C}^{2} \rightarrow \mathbb{P}^{n}$ is

$\nabla_{\boldsymbol{\lambda}}=\mathrm{d}+\left(\begin{array}{cc}\mathcal{A}_{11}(x, y) & \mathcal{A}_{12}(x, y) \\ -\mathcal{A}_{21}(x, y) & -\mathcal{A}_{11}(x, y)\end{array}\right)+\sum_{i=1}^{n-2}\left(\begin{array}{cc}\mathcal{A}_{11}^{i}\left(x, y, z_{i}\right) & \mathcal{A}_{12}^{i}\left(x, y, z_{i}\right) \\ -\mathcal{A}_{21}^{i}\left(x, y, z_{i}\right) & -\mathcal{A}_{11}^{i}\left(x, y, z_{i}\right)\end{array}\right)$,

whose polar divisor is $D_{n}$. This connection $\nabla_{\boldsymbol{\lambda}}$ is the connection described in Section 1.1. We consider a relation between $\nabla_{\boldsymbol{\lambda}}$ and $\left(\nabla_{1}\right)_{\boldsymbol{\lambda}}$. Let $\nabla_{1}^{\prime}$ be the meromorphic connection on $\mathbb{P}^{n} \times \mathbb{C} \rightarrow \mathbb{P}^{n}$ defined by $\nabla_{1}^{\prime}:=\mathrm{d}-\frac{1}{2} \frac{\mathrm{d} y}{y}$. We 
define a matrix $M_{2}(x, y)$ on $Y$ by

$$
M_{2}(x, y):=\left(\begin{array}{cc}
y & x-1 \\
0 & 1
\end{array}\right) .
$$

Let $\nabla_{1}^{\prime \prime}$ be the meromorphic connection on $\mathbb{P}^{n} \times \mathbb{C}^{2} \rightarrow \mathbb{P}^{n}$ defined by

$$
\begin{aligned}
\nabla_{1}^{\prime \prime}=\mathrm{d}+ & M_{2}(x, y)^{-1} \mathrm{~d} M_{2}(x, y) \\
& +M_{2}(x, y)^{-1}\left(\begin{array}{cc}
\alpha_{1}(x, y) & \alpha_{0}(x, y) \\
-\alpha_{2}(x, y) & -\alpha_{1}(x, y)
\end{array}\right) M_{2}(x, y) \\
& +\sum_{i=1}^{n-2} M_{2}(x, y)^{-1}\left(\begin{array}{cc}
0 & \alpha_{0}^{i}\left(x, y, z_{i}\right) \\
-\alpha_{2}^{i}\left(x, y, z_{i}\right) & 0
\end{array}\right) M_{2}(x, y) .
\end{aligned}
$$

We can check that

$$
\nabla_{\boldsymbol{\lambda}}=\nabla_{1}^{\prime \prime} \otimes \nabla_{1}^{\prime}
$$

By a combination of the equalities (2.4) and (2.8), we have the following proposition:

Proposition 2.2. - The pull-back $f^{*} \nabla_{\boldsymbol{\lambda}}$ is birationally equivalent to $\left(\nabla_{0}\right)_{\boldsymbol{\lambda}} \otimes \nabla_{0}^{\prime} \otimes f^{*} \nabla_{1}^{\prime}$.

Proof of Theorem 1.1 and Theorem 1.2. - First, since $\left(\nabla_{0}\right)_{\boldsymbol{\lambda}}, \nabla_{0}^{\prime}$ and $\nabla_{1}^{\prime}$ are flat and $f$ is a generically finite Galois morphism, we have the flatness of $\nabla_{\boldsymbol{\lambda}}$ for each $\boldsymbol{\lambda}$ by Proposition 2.2. Second, we have that $\nabla_{\boldsymbol{\lambda}}$ has at worst regular singularities for each $\boldsymbol{\lambda}$ by the explicit expression of $\nabla_{\boldsymbol{\lambda}}$ and [4, Chapter II, Theorem 4.1(ii)]. Finally, the assertion of Theorem 1.2 is deduced by Proposition 2.2.

\section{Monodromy representation}

In this section, we consider the monodromy representation $\pi_{1}\left(\mathbb{P}^{n} \backslash D_{n}, *\right) \rightarrow$ $\mathrm{SL}_{2}(\mathbb{C})$ of $\nabla_{\boldsymbol{\lambda}}$ for generic $\boldsymbol{\lambda}$. In Section 3.1 and Section 3.2, we discuss structure of the fundamental group $\pi_{1}\left(\mathbb{P}^{n} \backslash D_{n}, *\right)$ by using the Zariski's hyperplane section theorem and the Zariski-Van-Kampen method. In Section 3.3, we show Theorem 1.3 by using the results in Section 3.1 and Section 3.2.

\subsection{Zariski's hyperplane section theorem}

Let $H_{i}(i=1, \ldots, n-2)$ be the hyperplanes in $\mathbb{P}^{n}$ defined by

$$
H_{i}:=\left(z_{i}-a_{i} x-b_{i} y-c_{i} t=0\right) \quad i=1, \ldots, n-2 .
$$


Here $a_{i}, b_{i}$, and $c_{i}(i=1, \ldots, n-2)$ are generic complex numbers. For simplicity, we assume that $0<\left|a_{i}\right| \ll 1$ and $0<\left|b_{i}\right| \ll 1$. Let $f(x, y, t)$ be the following quadratic polynomial

$$
\begin{aligned}
f(x, y, t): & =x^{2}+y^{2}+t^{2}-2(x y+y t+t x) \\
& =(y-x-t)^{2}-4 x t .
\end{aligned}
$$

Let $\widetilde{\mathcal{Q}}_{0}, \widetilde{\mathcal{Q}}_{i}$ and $\widetilde{D}_{n}$ be the divisors on $\mathbb{P}^{2}=\mathbb{P}^{n} \cap\left(\bigcap_{i=1}^{n-2} H_{i}\right)$ defined by

$$
\begin{aligned}
\widetilde{\mathcal{Q}}_{0} & :=(f(x, y, t)=0), \\
\widetilde{\mathcal{Q}}_{i} & :=\left(f(x, y, t)-\left(a_{i} x+b_{i} y+c_{i} t\right)^{2}=0\right) \quad(i=1, \ldots, n-2), \text { and } \\
\widetilde{D}_{n} & :=(x=0)+(y=0)+(t=0)+\widetilde{\mathcal{Q}}_{0}+\widetilde{\mathcal{Q}}_{1}+\cdots+\widetilde{\mathcal{Q}}_{n-2},
\end{aligned}
$$

respectively. By Zariski's hyperplane section theorem (for example see [8]), we have the natural isomorphism

$$
\pi_{1}\left(\mathbb{P}^{n} \backslash D_{n}, *\right) \cong \pi_{1}\left(\mathbb{P}^{2} \backslash \widetilde{D}_{n}, *\right) .
$$

\subsection{Zariski-Van-Kampen method}

We derive some equalities in $\pi_{1}\left(\mathbb{P}^{2} \backslash \widetilde{D}_{n}, *\right)$ by the Zariski-Van-Kampen method (see for example [3]).

Let $\pi: \mathbb{P}^{2} \backslash \widetilde{D}_{n} \rightarrow \mathbb{P}^{1}$ be the projection defined by

$$
\begin{aligned}
\pi: \mathbb{P}^{2} \backslash \widetilde{D}_{n} & \longrightarrow \mathbb{P}^{1} \\
{[x: y: t] } & \longmapsto[x: t] .
\end{aligned}
$$

Let $\left\{\left[x_{i}^{+}: 1\right],\left[x_{i}^{-}: 1\right]\right\} \subset \mathbb{P}^{1}$ be the roots of the discriminant of $f(x, y, t)-$ $\left(a_{i} x+b_{i} y+c_{i} t\right)^{2}$ with respect to $y$. We denote $\left[x_{i}^{+}: 1\right]$ and $\left[x_{i}^{-}: 1\right]$ by $x_{i}^{+}$and $x_{i}^{-}$, respectively. Since $0<\left|a_{i}\right| \ll 1$ and $0<\left|b_{i}\right| \ll 1$, there exists an element of $\left\{x_{i}^{+}, x_{i}^{-}\right\}$in a neighborhood of $\infty=[0: 1]$. We assume that $x_{i}^{-}$is a point in a neighborhood of $\infty$. Set $a=[a: 1]$ where $0<|a| \ll 0$. For $i=0,1, \ldots, n-2$, let $y_{i}^{+}$and $y_{i}^{-}$be the intersection of $\widetilde{\mathcal{Q}}_{i}$ and $\pi^{-1}(a)$ : $\widetilde{\mathcal{Q}}_{i} \cap \pi^{-1}(a)=\left\{y_{i}^{+}, y_{i}^{-}\right\}$. Here we assume that

$$
0<\operatorname{Arg}\left(\frac{y_{1}^{+}-(a+1)}{y_{0}^{+}-(a+1)}\right)<\cdots<\operatorname{Arg}\left(\frac{y_{n-2}^{+}-(a+1)}{y_{0}^{+}-(a+1)}\right)<\pi .
$$




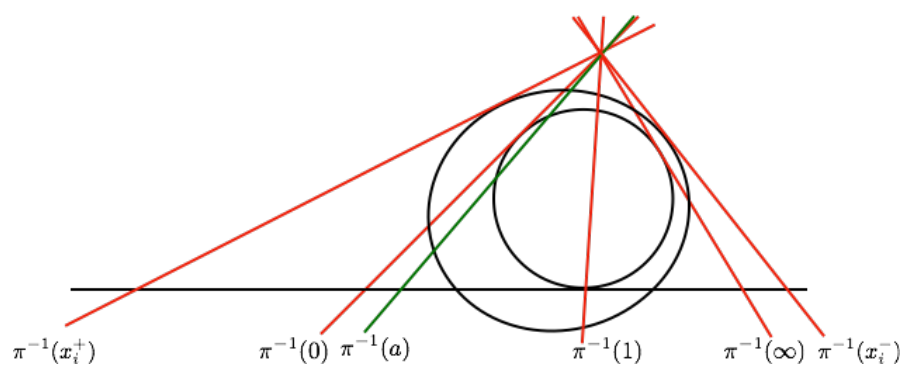

Figure 3.1. Fibers of $\pi$.

We define natural numbers $i_{k}$ and $j_{k}(k=1, \ldots, n-2)$ so that $\left\{i_{1}, \ldots, i_{n-2}\right\}=\{1, \ldots, n-2\},\left\{j_{1}, \ldots, j_{n-2}\right\}=\{1, \ldots, n-2\}$,

$$
\begin{aligned}
0<\operatorname{Arg}\left(x_{i_{1}}^{+}\right)<\cdots<\operatorname{Arg}\left(x_{i_{n-2}}^{+}\right) & <2 \pi \\
\text { and } 0 & <\operatorname{Arg} \frac{1}{x_{j_{1}}^{-}}<\cdots<\operatorname{Arg} \frac{1}{x_{j_{n-2}}^{-}}<2 \pi .
\end{aligned}
$$

Here we define the range of the principal value of arguments Arg by the closed-open interval $[0,2 \pi)$. Let $\Gamma$ be the group defined by

$$
\Gamma:=\left\langle\begin{array}{c}
\alpha_{0}, \alpha_{y_{0}^{+}}, \ldots, \alpha_{y_{n-2}^{+}} \\
\alpha_{y_{0}^{-}}, \ldots, \alpha_{y_{n-2}^{-}}, \alpha_{\infty}
\end{array} \mid \alpha_{0} \alpha_{y_{1}^{+}} \cdots \alpha_{y_{n-2}^{+}} \alpha_{y_{0}^{-}} \alpha_{y_{1}^{-}} \ldots \alpha_{y_{n-2}^{-}} \alpha_{y_{0}^{+}} \alpha_{\infty}=1\right\rangle .
$$

Then we have $\pi_{1}\left(\pi^{-1}(a) \backslash\left(\widetilde{D}_{n} \cap \pi^{-1}(a)\right), *\right) \cong \Gamma$ and have an exact sequence

$$
1 \longrightarrow \Gamma \longrightarrow \pi_{1}\left(\mathbb{P}^{2} \backslash \widetilde{D}_{n}, *\right) \longrightarrow \pi_{1}\left(\mathbb{P}^{1} \backslash\{0, \infty\}, a\right) \longrightarrow 1 .
$$

Let

$$
\gamma_{0}, \gamma_{1}, \gamma_{x_{1}^{+}}, \ldots, \gamma_{x_{n-2}^{+}}, \gamma_{x_{1}^{-}}, \ldots, \gamma_{x_{n-2}^{-}}, \gamma_{\infty}
$$

be loops with base point $a$ on $\mathbb{P}^{1} \backslash\left\{0,1, x_{1}^{ \pm}, \ldots, x_{n-1}^{ \pm}, \infty\right\}$ such that for $x \in\left\{0,1, x_{1}^{ \pm}, \ldots, x_{n-1}^{ \pm}, \infty\right\}$, the loop $\gamma_{x}$ is oriented counter-clockwise, $x$ lies inside, while the other points $\left\{0,1, x_{1}^{ \pm}, \ldots, x_{n-1}^{ \pm}, \infty\right\} \backslash\{x\}$ lie outside as in Figure 3.2.

Let $s: \mathbb{P}^{1} \backslash\{0, \infty\} \rightarrow \mathbb{P}^{2} \backslash \widetilde{D}_{n}$ be a continuous section of $\pi$ such that $s(a)=$ $* \in \mathbb{P}^{2} \backslash \widetilde{D}_{n}$. For the loops (3.1), we define the monodromy actions of the loops (3.1) on $\Gamma$ as in [2, Theorem 2.2.1]. Namely, the action $\left(\gamma_{x}, \alpha\right) \mapsto \gamma_{x}(\alpha)$ for loops $\gamma_{x}$ and $\alpha \in \Gamma$ is characterized by the equality $\gamma_{x}(\alpha)=\gamma_{x}^{-1} \alpha \gamma_{x}$ in $\pi_{1}\left(\mathbb{P}^{2} \backslash \widetilde{D}_{n}, *\right)$. Here we denote by $\gamma_{x}$ the loop $s_{*}\left(\gamma_{x}\right) \in \pi_{1}\left(\mathbb{P}^{2} \backslash \widetilde{D}_{n}, *\right)$ for simplicity. For explicit computation of this action, we consider the motion of the points $y_{i}^{ \pm}(i=0,1, \ldots, n-2)$ when $a$ varies along the loop $\gamma_{x}$ and a continuous deformation of $\alpha \in \pi_{1}\left(\pi^{-1}(a) \backslash\left(\widetilde{D}_{n} \cap \pi^{-1}(a)\right)\right.$, *) according 


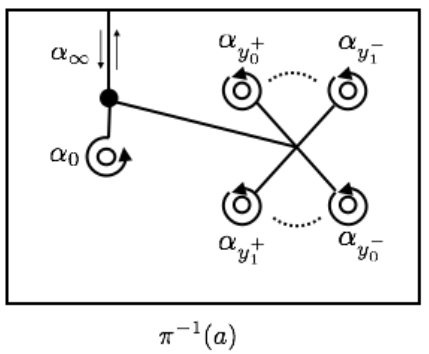

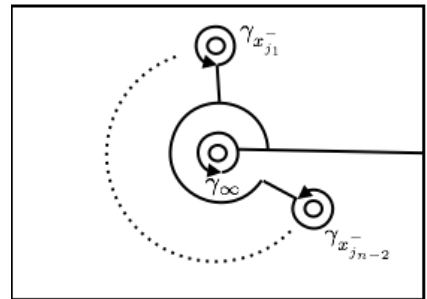

$\left(\mathbb{C}_{x}\right)_{\infty}$

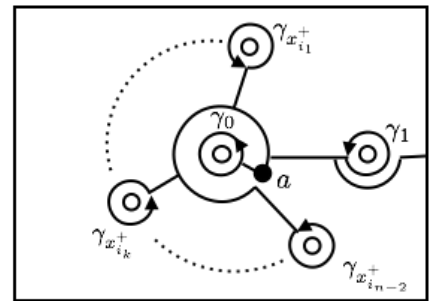

$\left(\mathbb{C}_{x}\right)_{0}$

Figure 3.2. Loops on $\pi^{-1}(a)$ and $\mathbb{P}^{1}=\left(\mathbb{C}_{x}\right)_{0} \cup\left(\mathbb{C}_{x}\right)_{\infty}$.

to the motion of these points. Note that the assumptions $0<\left|a_{i}\right| \ll 1$, $0<\left|b_{i}\right| \ll 1$ and $0<|a| \ll 1$ make the computation of the motion of the points $y_{i}^{ \pm}$simple. By explicit computation of the action on some loops, we can check the following equalities:

$$
\begin{aligned}
\gamma_{0}\left(\alpha_{y_{0}^{+}}\right) & =\alpha_{y_{0}^{-}} ; & \gamma_{x_{i}^{+}}\left(\alpha_{y_{i}^{+}}\right) & =\alpha_{y_{i}^{+}} \alpha_{y_{i}^{-}} \alpha_{y_{i}^{+}}^{-1}(i=1, \ldots, n-2) ; \\
\gamma_{0}\left(\alpha_{0}\right) & =\alpha_{0} ; & \gamma_{0}\left(\alpha_{y_{i}^{+}}\right) & =\alpha_{y_{i}^{+}}(i=1, \ldots, n-2) ; \\
\widetilde{\gamma}_{\infty}\left(\alpha_{y_{0}^{+}}\right) & =\alpha_{0} \alpha_{y_{0}^{-}} \alpha_{0}^{-1} ; & &
\end{aligned}
$$

and

$$
\gamma_{1}\left(\alpha_{y_{0}^{+}}\right)=\left(\alpha_{0} \alpha_{y_{0}^{+}}\right) \alpha_{0} \alpha_{y_{0}^{+}} \alpha_{0}^{-1}\left(\alpha_{0} \alpha_{y_{0}^{+}}\right)^{-1} .
$$

Here we put $\widetilde{\gamma}_{\infty}:=\gamma_{x_{j_{1}}^{-}} \cdots \gamma_{x_{j_{n-2}}^{-}} \gamma_{\infty}$. In fact, if $a$ varies along the loop $\gamma_{0}$, then $y_{0}^{+}$moves to the location of $y_{0}^{-}$, and 0 and $y_{i}^{+}(i=1, \ldots, n-2)$ go back to the prior locations, respectively. If $a$ varies along the loop $\gamma_{x_{i}^{+}}$for $i=1, \ldots, n-2$, then $y_{i}^{+}$moves to the location of $y_{i}^{-}$. Here we assume that $-1 \ll \operatorname{Arg}(a)<0$ and $y_{0}^{+}$closes to 0 when $a$ approach 1 along the real axis. If $a$ varies along the loop $\widetilde{\gamma}_{\infty}$, then $y_{0}^{+}$moves to the location of $y_{0}^{-}$round by 0 . If $a$ varies along the loop $\gamma_{1}$, then $y_{0}^{+}$go back to the prior locations round by 0 twice. If we consider continuous deformations of the corresponding loops according to the motion of these points, then we have the equalities (3.2), (3.3), (3.4) and (3.5). Here note that the images of the intersection of $\mathcal{Q}_{i}$ 
and $\mathcal{Q}_{j}$ under $\pi$ are close to $\infty$ for $i, j=0,1, \ldots, n-2$ since $0<\left|a_{i}\right| \ll 1$ and $0<\left|b_{i}\right| \ll 1$.

In $\pi_{1}\left(\mathbb{P}^{2} \backslash \widetilde{D}_{n}, *\right)$, we have the equality $\gamma_{x}(\alpha)=\gamma_{x}^{-1} \alpha \gamma_{x}$ for $\alpha \in \Gamma$. By the equality $(3.2)$, we can show that $\alpha_{y_{i}^{-}}(i=0, \ldots, n-2)$ are generated by $\alpha_{y_{0}^{+}}, \ldots, \alpha_{y_{n-2}^{+}}$, and $\gamma_{0}$ in $\pi_{1}\left(\mathbb{P}^{2} \backslash \widetilde{D}_{n}, *\right)$. Then we obtain the following proposition.

Proposition 3.1. - The group $\pi_{1}\left(\mathbb{P}^{2} \backslash \widetilde{D}_{n}, *\right)$ is generated by $\alpha_{0}, \alpha_{y_{0}^{+}}$, $\ldots, \alpha_{y_{n-2}^{+}}$, and $\gamma_{0}$.

Proposition 3.2. - Set $\widetilde{\alpha}=\alpha_{y_{1}^{+}} \cdots \alpha_{y_{n-2}^{+}}$. For the elements $\alpha_{y_{0}^{+}}, \alpha_{0}, \gamma_{0}$, and $\widetilde{\alpha}$ of $\pi_{1}\left(\mathbb{P}^{2} \backslash \widetilde{D}_{n}, *\right)$, we have the following equalities:

$$
\begin{aligned}
{\left[\alpha_{0}, \gamma_{0}\right] } & =\left[\widetilde{\alpha}, \gamma_{0}\right]=1, \\
\left(\left(\gamma_{0} \widetilde{\alpha}\right) \alpha_{y_{0}^{+}}\right)^{2} & =\left(\alpha_{y_{0}^{+}}\left(\gamma_{0} \widetilde{\alpha}\right)\right)^{2}, \\
\left(\alpha_{y_{0}^{+}} \alpha_{0}\right)^{2} & =\left(\alpha_{0} \alpha_{y_{0}^{+}}\right)^{2} .
\end{aligned}
$$

Proof. - By the equality (3.3), we have the equality (3.6). Second, we show the equality (3.7). By the equalities (3.2), (3.4), $a_{\infty}=\gamma_{\infty} \gamma_{0}$, and (3.6), we have

$$
\begin{aligned}
a_{y_{0}^{+}}= & \gamma_{\infty} \alpha_{0} \gamma_{0}^{-1} \alpha_{y_{0}^{+}} \gamma_{0} \alpha_{0}^{-1} \gamma_{\infty}^{-1} \\
= & \alpha_{\infty} \alpha_{0} \gamma_{0}^{-1} \gamma_{0}^{-1} \alpha_{y_{0}^{+}} \gamma_{0} \gamma_{0} \alpha_{0}^{-1} \alpha_{\infty}^{-1} \\
= & \left(\alpha_{y_{1}^{+}} \cdots \alpha_{y_{n-2}^{+}} \alpha_{y_{0}^{-}} \alpha_{y_{1}^{-}} \cdots \alpha_{y_{n-2}^{-}} \alpha_{y_{0}^{+}}\right)^{-1} \\
& \quad \times \gamma_{0}^{-1} \gamma_{0}^{-1} \alpha_{y_{0}^{+}} \gamma_{0} \gamma_{0}\left(\alpha_{y_{1}^{+}} \cdots \alpha_{y_{n-2}^{+}} \alpha_{y_{0}^{-}} \alpha_{y_{1}^{-}} \cdots \alpha_{y_{n-2}^{-}} \alpha_{y_{0}^{+}}\right) \\
= & \left(\widetilde{\alpha} \gamma_{0}^{-1} \alpha_{y_{0}^{+}} \gamma_{0} \widetilde{\alpha} \alpha_{y_{0}^{+}}\right)^{-1} \gamma_{0}^{-1} \gamma_{0}^{-1} \alpha_{y_{0}^{+}} \gamma_{0} \gamma_{0}\left(\widetilde{\alpha} \gamma_{0}^{-1} \alpha_{y_{0}^{+}} \gamma_{0} \widetilde{\alpha} \alpha_{y_{0}^{+}}\right) \\
= & \left(\widetilde{\alpha} \alpha_{y_{0}^{+}} \gamma_{0} \widetilde{\alpha} \alpha_{y_{0}^{+}}\right)^{-1} \gamma_{0}^{-1} \alpha_{y_{0}^{+}} \gamma_{0}\left(\widetilde{\alpha} \alpha_{y_{0}^{+}} \gamma_{0} \widetilde{\alpha} \alpha_{y_{0}^{+}}\right) .
\end{aligned}
$$

Then we have the equality (3.7). By the equality (3.5), we have the equality (3.8).

\subsection{Monodromy representation of $\nabla_{\lambda}$}

Let $\boldsymbol{D}_{\infty}$ be the infinite dihedral group:

$$
\boldsymbol{D}_{\infty}:=\left\langle\left(\begin{array}{cc}
0 & \alpha \\
-\alpha^{-1} & 0
\end{array}\right),\left(\begin{array}{cc}
\beta & 0 \\
0 & \beta^{-1}
\end{array}\right) \mid \alpha, \beta \in \mathbb{C}^{*}\right\rangle \leqslant \mathrm{SL}_{2}(\mathbb{C}) .
$$


PROPOSITION 3.3. - For generic $\boldsymbol{\lambda}$, the monodromy representation of $\nabla_{\boldsymbol{\lambda}}$ is conjugated to the dihedral representation $\rho_{\boldsymbol{\lambda}}: \pi_{1}\left(\mathbb{P}^{n} \backslash D_{n}, *\right) \rightarrow \boldsymbol{D}_{\infty}$ of the fundamental group $\pi_{1}\left(\mathbb{P}^{n} \backslash D_{n}, *\right)$ defined by

$$
\begin{aligned}
\rho_{\boldsymbol{\lambda}}\left(\alpha_{0}\right) & =\left(\begin{array}{cc}
-\exp \left(-\pi \lambda_{0}\right) & 0 \\
0 & -\exp \left(\pi \lambda_{0}\right)
\end{array}\right), \\
\rho_{\boldsymbol{\lambda}}\left(\gamma_{0}\right) & =\left(\begin{array}{cc}
\exp \left(-\pi \lambda_{1}\right) & 0 \\
0 & \exp \left(\pi \lambda_{1}\right)
\end{array}\right), \\
\rho_{\boldsymbol{\lambda}}\left(\alpha_{y_{0}^{+}}\right) & =\left(\begin{array}{cc}
0 & 1 \\
-1 & 0
\end{array}\right), \\
\rho_{\boldsymbol{\lambda}}\left(\alpha_{y_{i}^{+}}\right) & =\left(\begin{array}{cc}
\exp \left(-\pi \lambda_{i+1}\right) & 0 \\
0 & \exp \left(\pi \lambda_{i+1}\right)
\end{array}\right),
\end{aligned}
$$

where $i=1, \ldots, n-2$.

Proof. - Let $\rho_{\nabla_{\lambda}}: \pi_{1}\left(\mathbb{P}^{n} \backslash D_{n}, *\right) \rightarrow \mathrm{SL}_{2}(\mathbb{C})$ be a monodromy representation of $\nabla_{\boldsymbol{\lambda}}$. Put $A_{0}:=\rho_{\nabla_{\boldsymbol{\lambda}}}\left(\alpha_{0}\right), A_{y_{i}^{+}}:=\rho_{\nabla_{\boldsymbol{\lambda}}}\left(\alpha_{y_{i}^{+}}\right)(i=0, \ldots, n-1)$ and $C_{0}:=\rho_{\nabla_{\boldsymbol{\lambda}}}\left(\gamma_{0}\right)$. Let $U$ be some analytic open subset of $\mathbb{P}^{2} \backslash\left(\widetilde{\mathcal{Q}}_{0} \cup(y=0) \cup\right.$ $(t=0))$ such that $U$ is simply connected and $U$ contains the loops $\alpha_{y_{i}^{+}}$ $(i=1, \ldots, n-1)$ and $\gamma_{0}$. On the open subset $U$, the connection $\nabla_{\boldsymbol{\lambda}}$ is isomorphic to $\left(\nabla_{0}\right)_{\lambda}$. Then by some conjugation, we may put

$$
\begin{aligned}
C_{0}=\left(\begin{array}{cc}
\exp \left(-\pi \lambda_{1}\right) & 0 \\
0 & \exp \left(\pi \lambda_{1}\right)
\end{array}\right), \\
A_{y_{i}^{+}}=\left(\begin{array}{cc}
\exp \left(-\pi \lambda_{i+1}\right) & 0 \\
0 & \exp \left(\pi \lambda_{i+1}\right)
\end{array}\right) \quad i=1, \ldots, n-2 .
\end{aligned}
$$

Assume that $\exp \left(-\pi \lambda_{1}\right) \neq \exp \left(\pi \lambda_{1}\right)$. By Proposition 3.2, we have the equality $A_{0} C_{0}=C_{0} A_{0}$. Then we have

$$
A_{0}=\left(\begin{array}{cc}
-\exp \left(-\pi \lambda_{0}\right) & 0 \\
0 & -\exp \left(\pi \lambda_{0}\right)
\end{array}\right) .
$$

Note that the image $\operatorname{Im}\left(\rho_{\nabla_{\lambda}}\right)$ is non-abelian. Since $C_{0}, A_{0}$, and $A_{y_{i}^{+}}$ $(i=1, \ldots, n-2)$ are diagonal matrices, we may put

$$
A_{y_{0}^{+}}=\left(\begin{array}{cc}
a_{11} & a_{12} \\
-1 & a_{22}
\end{array}\right) \text {. }
$$

Put $\tilde{A}:=A_{y_{1}^{+}} \cdots A_{y_{n-2}^{+}}$. By Proposition 3.2 , we have the equalities $\left(A_{y_{0}^{+}}\left(C_{0} \widetilde{A}\right)\right)^{2}=\left(\left(C_{0} \widetilde{A}\right) A_{y_{0}^{+}}\right)^{2}$ and $\left(A_{y_{0}^{+}} A_{0}\right)^{2}=\left(A_{0} A_{y_{0}^{+}}\right)^{2}$. Assume that $\left(\exp \left(-\pi \lambda_{1}-\pi \sum_{i=1}^{n-2} \lambda_{i+1}\right)\right)^{2} \neq 1$ and $\left(-\exp \left(-\pi \lambda_{0}\right)\right)^{2} \neq 1$. Since $A_{y_{0}^{+}}\left(C_{0} \widetilde{A}\right) \neq$ $\left(C_{0} \widetilde{A}\right) A_{y_{0}^{+}}$and $A_{y_{0}^{+}} A_{0} \neq A_{0} A_{y_{0}^{+}}$, we have the equalities $\left(A_{y_{0}^{+}}\left(C_{0} \widetilde{A}\right)\right)^{2}=-I_{2}$ 
and $\left(A_{y_{0}^{+}} A_{0}\right)^{2}=-I_{2}$. Then we have the following equalities:

$$
\left\{\begin{array}{l}
a_{11} a_{22}+a_{12}=1 \\
a_{11}\left(\exp \left(-\pi \lambda_{1}-\pi \sum_{i=1}^{n-2} \lambda_{i+1}\right)\right)^{2}=a_{22} \\
a_{11}\left(-\exp \left(-\pi \lambda_{0}\right)\right)^{2}=a_{22} .
\end{array}\right.
$$

We assume that $\left(\exp \left(-\pi \lambda_{1}-\pi \sum_{i=1}^{n-2} \lambda_{i+1}\right)\right)^{2} \neq\left(-\exp \left(-\pi \lambda_{0}\right)\right)^{2}$. Then we have $a_{11}=0, a_{12}=1$, and $a_{22}=0$.

Proof of Theorem 1.3. - By Theorem 1.2, we have that the monodromy representation of $\nabla_{\boldsymbol{\lambda}}$ is virtually abelian. By Proposition 3.3, we have that the monodromy representation of $\nabla_{\boldsymbol{\lambda}}$ is conjugated to the explicit representation

$$
\rho_{\boldsymbol{\lambda}}: \pi_{1}\left(\mathbb{P}^{n} \backslash D_{n}\right) \longrightarrow \mathrm{SL}_{2}(\mathbb{C}),
$$

which takes values in the infinite dihedral group $\boldsymbol{D}_{\infty}$.

\section{Algebraic Garnier solution}

Assume that an $n$-tuple of complex numbers $\boldsymbol{\lambda}=\left(\lambda_{0}, \ldots, \lambda_{n-1}\right)$ is sufficiently generic. In this section, we restrict the flat connection $\nabla_{\boldsymbol{\lambda}}$ to a generic line $\mathbb{P}^{n} \cap\left(\bigcap_{i=0}^{n-2} H_{i}^{\prime}\right)$, where

$$
\left\{\begin{array}{l}
H_{0}^{\prime}=(y-a x-b t=0) \\
H_{i}^{\prime}=\left(z_{i}-c_{i} x-d_{i} t=0\right) \quad(i=1,2, \ldots, n-2) .
\end{array}\right.
$$

Here $a, b, c_{i}$, and $d_{i}(i=1,2, \ldots, n-2)$ are generic complex numbers. We consider the transformation $\widetilde{x}=-\frac{a}{b} x$. Let $T$ be a Zariski open subset of Spec $\mathbb{C}\left[a, b, c_{i}, d_{i}\right]_{i=1, \ldots, n-2}$. We consider the map $\mathbb{P}^{1} \times T \rightarrow \mathbb{P}^{n}$ defined by (4.1). Let $\left(\nabla_{\mathbb{P}^{1} \times T}\right)_{\boldsymbol{\lambda}}$ be the flat connection on the trivial rank 2 vector bundle $F_{0}$ over $\mathbb{P}^{1} \times T$ induced by the flat connection $\nabla_{\boldsymbol{\lambda}}$ over $\mathbb{P}^{n}$. Let

$$
\left(\nabla_{\mathbb{P}^{1} \times T / T}\right)_{\boldsymbol{\lambda}}: F_{0} \longrightarrow F_{0} \otimes \Omega_{\mathbb{P}^{1} \times T / T}^{1}\left(D_{n}\right)
$$

be the relative connection on $F_{0}$ over $\mathbb{P}^{1} \times T$ associated to $\left(\nabla_{\mathbb{P}^{1} \times T}\right)_{\boldsymbol{\lambda}}$. In Section 4.1 , we introduce an étale base change $\widetilde{T} \rightarrow T$ to prove the assertion (i) of Theorem 1.4. In Section 4.2, after the étale base change $\widetilde{T} \rightarrow T$, we compute the residue matrix of $\left(\nabla_{\mathbb{P}^{1} \times \tilde{T} / \tilde{T}}\right)_{\boldsymbol{\lambda}}$ for each simple pole. In Section 4.3, we recall the relation between isomonodromic deformations and the Garnier system following [10]. In Section 4.4, we show Theorem 1.4. 


\subsection{Regular singular points of $\left(\nabla_{\mathbb{P}^{1} \times T / T}\right)_{\lambda}$}

By the pull-back of $x^{2}+y^{2}+t^{2}-2(x y+y t+t x)$ and $x^{2}+y^{2}+t^{2}-$ $2(x y+y t+t x)-z_{i}^{2}$ under $\mathbb{P}^{1} \times T \rightarrow \mathbb{P}^{n}$, we have the following polynomials over $T$ :

$$
\begin{aligned}
f(a, b, \widetilde{x}) & :=\frac{(a-1)^{2} b^{2}}{a^{2}} \widetilde{x}^{2}+\frac{2 b(1+a+b-a b)}{a} \widetilde{x}+(b-1)^{2}, \\
f_{i}\left(a, b, c_{i}, d_{i}, \widetilde{x}\right) & :=f(a, b, \widetilde{x})-\frac{\left(a d_{i}-b c_{i} \widetilde{x}\right)^{2}}{a^{2}},
\end{aligned}
$$

which are described on the affine coordinate $[\widetilde{x}: 1]$. Let $I$ be the ideal of $\mathbb{C}\left[a, b, \widetilde{b}, c_{i}, d_{i}, \widetilde{d}_{i}\right]_{i=1, \ldots, n-2}$ defined by $I:=\left(\widetilde{b}^{2}-4(a+b-a b), \widetilde{d}_{i}^{2}-\Delta_{\tilde{x}}^{i}\right)_{i=1, \ldots, n-2}$, where $\Delta_{\widetilde{x}}^{i}$ is the discriminant of $f_{i}\left(a, b, c_{i}, d_{i}, \widetilde{x}\right)$ with respect to $\widetilde{x}$. We have the natural morphism

$$
\operatorname{Spec} \mathbb{C}\left[a, b, \widetilde{b}, c_{i}, d_{i}, \widetilde{d}_{i}\right]_{i=1, \ldots, n-2} / I \longrightarrow \operatorname{Spec} \mathbb{C}\left[a, b, c_{i}, d_{i}\right]_{i=1, \ldots, n-2} .
$$

Let $\widetilde{T}$ be the inverse image of $T$ under this morphism: $\widetilde{T} \rightarrow T$. Let $t_{1}$ and $t_{2}$ be the rational functions on $\widetilde{T}$ defined by

$$
t_{1}:=\frac{a(\widetilde{b}-2)^{2}}{4(a-1)\left(\widetilde{b}^{2}-a\right)} \text { and } t_{2}:=\frac{a(\widetilde{b}+2)^{2}}{4(a-1)\left(\widetilde{b}^{2}-a\right)} .
$$

Then $f\left(a, b, t_{1}\right)=f\left(a, b, t_{2}\right)=0$. Moreover, let $t_{2 i+1}$ and $t_{2 i+2}$ be the rational functions on $\widetilde{T}$ defined by

$$
\begin{aligned}
t_{2 i+1} & :=\frac{2 a b\left(1+a+b-a b-c_{i} d_{i}\right)-a^{2} \widetilde{d}_{i}}{2 b^{2}\left((a-1)^{2}-c_{i}^{2}\right)} \\
\text { and } \quad t_{2 i+2} & :=\frac{2 a b\left(1+a+b-a b-c_{i} d_{i}\right)+a^{2} \widetilde{d}_{i}}{2 b^{2}\left((a-1)^{2}-c_{i}^{2}\right)} .
\end{aligned}
$$

Then $f_{i}\left(a, b, c_{i}, d_{i}, t_{2 i+1}\right)=f_{i}\left(a, b, c_{i}, d_{i}, t_{2 i+2}\right)=0$. By these rational functions, we have a generically finite morphism

$$
\widetilde{T} \longrightarrow \operatorname{Spec} \mathbb{C}\left[t_{1}, t_{2}, \ldots, t_{2 n-2}\right],
$$

if the Zariski open subset $T$ shrinks. We take the pull-back $\left(\nabla_{\mathbb{P}^{1} \times \tilde{T} / \tilde{T}}\right)_{\boldsymbol{\lambda}}$ of $\left(\nabla_{\mathbb{P}^{1} \times T / T}\right)_{\boldsymbol{\lambda}}$ under the morphism $\mathbb{P}^{1} \times \widetilde{T} \rightarrow \mathbb{P}^{1} \times T$. Then $\left(\nabla_{\mathbb{P}^{1} \times \tilde{T} / \tilde{T}}\right)_{\boldsymbol{\lambda}}$ is a family of the Fuchsian systems with $2 n+1$ regular singularities at $\widetilde{x}=$ $0,1, t_{1}, \ldots, t_{2 n-2}, \infty$ parametrized by $\widetilde{T}$. 


\subsection{Residue matrices of $\left(\nabla_{\mathbb{P}^{1} \times \tilde{T} / \tilde{T}}\right)_{\boldsymbol{\lambda}}$}

We describe the residue matrices of $\left(\nabla_{\mathbb{P}^{1} \times \tilde{T} / \tilde{T}}\right)_{\boldsymbol{\lambda}}$ at the regular singular points. Put

$$
M_{2}(\widetilde{x}):=\left(\begin{array}{cc}
-a b(\widetilde{x}-1) & -b \widetilde{x}-a \\
0 & a
\end{array}\right)
$$

and $\quad \alpha_{0}^{i}(\widetilde{x}):=\lambda_{i+1}\left(-\frac{b c_{i}}{a}-\frac{a d_{i}-b c_{i} \widetilde{x}}{2 a\left(\widetilde{x}-t_{2 i+1}\right)}-\frac{a d_{i}-b c_{i} \widetilde{x}}{2 a\left(\widetilde{x}-t_{2 i+2}\right)}\right)$.

Let $H_{2 n-1}^{\tilde{T}}$ be the residue matrix at $\widetilde{x}=0$. We have the following equality

$$
H_{2 n-1}^{\tilde{T}}=M_{2}(0)^{-1}\left(\begin{array}{cc}
0 & \frac{\lambda_{1}\left(\tilde{b}^{2}-4\right)}{8(a-1)} \\
\frac{2 \lambda_{1}(a-1)}{\tilde{b}^{2}-4} & 0
\end{array}\right) M_{2}(0) .
$$

Let $H_{2 n}^{\tilde{T}}$ be the residue matrix at $\widetilde{x}=1$. We have the following equality

$$
H_{2 n}^{\tilde{T}}=\frac{1-\lambda_{0}}{2}\left(\begin{array}{cc}
1 & \frac{2\left(\tilde{b}^{2}+4 a^{2}-8 a\right)}{\tilde{b}^{2}-4 a^{2}} \\
0 & -1
\end{array}\right)+\sum_{i=1}^{n-2} \alpha_{0}^{i}(1)\left(\begin{array}{cc}
0 & 1+\frac{(a+b)^{2}}{b^{2}(a-1)^{2}\left(1-t_{1}\right)\left(1-t_{2}\right)} \\
0 & 0
\end{array}\right) .
$$

Let $H_{1}^{\tilde{T}}$ and $H_{2}^{\tilde{T}}$ be the residue matrices at $\widetilde{x}=t_{1}$ and $\widetilde{x}=t_{2}$, respectively. We have the following equalities

$$
\begin{aligned}
& H_{1}^{\tilde{T}}=M_{2}\left(t_{1}\right)^{-1}\left(\begin{array}{cc}
-\frac{\lambda_{0}(a-1)}{2(\tilde{b}-2 a)}-\frac{\lambda_{1}(a-1)}{2(\tilde{b}-2)}+\sum_{i=1}^{n-2} \frac{a^{2} \alpha_{0}^{i}\left(t_{1}\right)}{b^{2}(a-1)^{2}\left(t_{1}-t_{2}\right)} & \frac{1}{4}
\end{array}\right) M_{2}\left(t_{1}\right) \\
& H_{2}^{\tilde{T}}=M_{2}\left(t_{2}\right)^{-1}\left(\begin{array}{cc}
-\frac{1}{4} & 0 \\
\frac{\lambda_{0}(a-1)}{2(\tilde{b}+2 a)}+\frac{\lambda_{1}(a-1)}{2(\tilde{b}+2)}+\sum_{i=1}^{n-2} \frac{a^{2} \alpha_{0}^{i}\left(t_{2}\right)}{b^{2}(a-1)^{2}\left(t_{2}-t_{1}\right)} & \frac{1}{4}
\end{array}\right) M_{2}\left(t_{2}\right) .
\end{aligned}
$$

Let $H_{2 i+1}^{\tilde{T}}$ and $H_{2 i+2}^{\tilde{T}}$ be the residue matrices at $\widetilde{x}=t_{2 i+1}$ and $\widetilde{x}=t_{2 i+2}$, respectively. We have the following equalities

$$
\begin{aligned}
& H_{2 i+1}^{\tilde{T}}=M_{2}\left(t_{2 i+1}\right)^{-1}\left(\begin{array}{cc}
0 & \frac{\lambda_{i+1}\left(a d_{i}-b c_{i} t_{2 i+1}\right)}{2 a} \\
\frac{\lambda_{i+1} a\left(-a d_{i}+b c_{i} t_{2 i+1}\right)}{2 b^{2}(a-1)^{2}\left(t_{2 i+1}-t_{1}\right)\left(t_{2 i+1}-t_{2}\right)} & 0
\end{array}\right) M_{2}\left(t_{2 i+1}\right) \\
& H_{2 i+2}^{\tilde{T}}=M_{2}\left(t_{2 i+2}\right)^{-1}\left(\begin{array}{cc}
0 & \frac{\lambda_{i+1}\left(a d_{i}-b c_{i} t_{2 i+2}\right)}{2 a} \\
\frac{\lambda_{i+1} a\left(-a d_{i}+b c_{i} t_{2 i+2}\right)}{2 b^{2}(a-1)^{2}\left(t_{2 i+2}-t_{1}\right)\left(t_{2 i+2}-t_{2}\right)} & 0
\end{array}\right) M_{2}\left(t_{2 i+2}\right) .
\end{aligned}
$$

Let $H_{2 n+1}^{\tilde{T}}$ be the residue matrix at $\widetilde{x}=\infty$. Let $\mathcal{A}_{j k}^{i}(\widetilde{x})$ be the relative rational 1-forms over $\widetilde{T}$ which are the relativization of the pull-backs of the rational 1-forms (2.7) under the composition $\mathbb{P}^{1} \times \widetilde{T} \rightarrow \mathbb{P}^{1} \times T \rightarrow \mathbb{P}^{n}$. Since $\lim _{\tilde{x} \rightarrow 0} \alpha_{0}^{i}\left(\frac{1}{\tilde{x}}\right)=0$, we have

$$
\operatorname{res}_{\tilde{x}=\infty}\left(\begin{array}{cc}
\mathcal{A}_{11}^{i}(\widetilde{x}) & \mathcal{A}_{12}^{i}(\widetilde{x}) \\
-\mathcal{A}_{21}^{i}(\widetilde{x}) & -\mathcal{A}_{11}^{i}(\widetilde{x})
\end{array}\right)=0 \quad(i=1, \ldots, n-2) .
$$


Then we have

$$
H_{2 n+1}^{\tilde{T}}=\left(\begin{array}{cc}
-1 & a-2 \\
1 & a
\end{array}\right)\left(\begin{array}{cc}
\frac{\lambda_{0}+\lambda_{1}}{2} & 0 \\
0 & -\frac{\lambda_{0}+\lambda_{1}}{2}
\end{array}\right)\left(\begin{array}{cc}
-1 & a-2 \\
1 & a
\end{array}\right)^{-1} .
$$

\subsection{Garnier system}

Let $\mathcal{A}(\widetilde{x})$ be the Fuchsian system with $2 n+1$ regular singularities at $t_{1}, \ldots, t_{2 n}, \infty$ :

$$
\mathcal{A}(\widetilde{x})=d+\sum_{i=1}^{2 n} \widetilde{H}_{i} \frac{\mathrm{d} \widetilde{x}}{\widetilde{x}-t_{i}},
$$

where $\widetilde{H}_{i}(i=1, \ldots, 2 n)$ are $2 \times 2$ matrices independent of $\widetilde{x}$ and $t_{i} \neq t_{j}$ $(i \neq j)$. We assume that $\widetilde{H}_{2 n+1}:=-\sum_{i=1}^{2 n} \widetilde{H}_{i}$ is a diagonal matrix and the eigenvalues of $\widetilde{H}_{i}(i=1, \ldots, 2 n+1)$ are as in Table 4.1 .

Table 4.1. The eigenvalues of the residue matrices $(i=1, \ldots, n-2)$.

\begin{tabular}{c|ccccccc} 
Reside matrices & $\widetilde{H}_{1}$ & $\widetilde{H}_{2}$ & $\widetilde{H}_{2 i+1}$ & $\widetilde{H}_{2 i+2}$ & $\widetilde{H}_{2 n-1}$ & $\widetilde{H}_{2 n}$ & $\widetilde{H}_{2 n+1}$ \\
\hline Eigenvalues & $\pm \frac{1}{4}$ & $\pm \frac{1}{4}$ & $\pm \frac{\lambda_{i+1}}{2}$ & $\pm \frac{\lambda_{i+1}}{2}$ & $\pm \frac{\lambda_{1}}{2}$ & $\pm \frac{\lambda_{0}-1}{2}$ & $\pm \frac{\lambda_{0}+\lambda_{1}}{2}$
\end{tabular}

We fix generators $\gamma_{\tilde{x}}\left(\widetilde{x}=t_{1} \ldots, t_{2 n}, \infty\right)$ of the fundamental group $\pi_{1}\left(\mathbb{P}^{1} \backslash\right.$ $\left.\left\{t_{1}, \ldots, t_{2 n}, \infty\right\}, *\right)$. Here the loop $\gamma_{\tilde{x}}$ on $\mathbb{P}^{1}$ is oriented counter-clockwise, $\widetilde{x}$ lies inside, while the other singular points lie outside. Let $\rho_{\lambda}^{\prime}: \pi_{1}\left(\mathbb{P}^{1} \backslash\right.$ $\left.\left\{t_{1}, \ldots, t_{2 n}, \infty\right\}, *\right) \rightarrow \mathrm{SL}_{2}(\mathbb{C})$ be the representation of the fundamental group defined by Table 4.2. We consider the isomonodromic deformation of the Fuchsian system $\mathcal{A}(\widetilde{x})$ whose preserved monodromy representation is conjugated to $\rho_{\boldsymbol{\lambda}}^{\prime}$. Let $d+\sum_{i=1}^{2 n} \widetilde{H}_{i}^{0} \frac{\mathrm{d} \tilde{x}}{\tilde{x}-t_{i}^{0}}$ be the Fuchsian system with $2 n+1$ regular singularities at $t_{1}^{0}, \ldots, t_{2 n}^{0}, \infty$ whose monodromy representation is conjugated to $\rho_{\lambda}^{\prime}$. There exists an open neighbourhood $U_{t^{0}} \subset \mathbb{C}^{2 n}$ of the point $t^{0}=\left(t_{1}^{0}, \ldots, t_{2 n}^{0}\right)$ such that for any $t \in U_{t^{0}}$, there exists a unique tuple $\left(\widetilde{H}_{i}(t)\right)_{i=1, \ldots, 2 n}$ of analytic matrix valued functions such that $\widetilde{H}_{i}\left(t^{0}\right)=\widetilde{H}_{i}^{0}$, $i=1, \ldots, 2 n$, and the monodromy representation of $d+\sum_{i=1}^{2 n} \widetilde{H}_{i}(t) \frac{\mathrm{d} \tilde{x}}{\tilde{x}-t_{i}}$ is conjugated to $\rho_{\boldsymbol{\lambda}}^{\prime}$. The matrices $\widetilde{H}_{i}(t) i=1, \ldots, 2 n$ are the solutions of the Cauchy problem with the initial data $\left(\widetilde{H}_{i}\left(t^{0}\right)\right)_{i=1, \ldots, 2 n}$ for the Schlesinger equations (see [10, Theorem 2.7]).

Let $\mathcal{A}(\widetilde{x})$ be the Fuchsian system with $2 n+1$ regular singularities at $t_{1}, \ldots, t_{2 n}, \infty$ as above. We fix the poles $t_{2 n-1}$ and $t_{2 n}$ at 0 and 1 , respectively. 
Table 4.2. The representation $\rho_{\boldsymbol{\lambda}}^{\prime}$ of the fundamental group; here $a_{j}=$ $\exp \left(-\pi \sqrt{-1} \lambda_{j}\right) j=0,1, \ldots, n-1$.

$$
\begin{aligned}
& \begin{array}{c|c|c}
\tilde{x}=t_{2 n-1} & \tilde{x}=t_{2 n} & \tilde{x}=t_{1} \\
\hline \rho_{\boldsymbol{\lambda}}^{\prime}\left(\gamma_{t_{2 n-1}}\right)=\left(\begin{array}{cc}
a_{1} & 0 \\
0 & a_{1}^{-1}
\end{array}\right) & \rho_{\boldsymbol{\lambda}}^{\prime}\left(\gamma_{t_{2 n}}\right)=\left(\begin{array}{cc}
-a_{0} & 0 \\
0 & -a_{0}^{-1}
\end{array}\right) & \rho_{\boldsymbol{\lambda}}^{\prime}\left(\gamma_{t_{1}}\right)=\left(\begin{array}{cc}
0 & 1 \\
-1 & 0
\end{array}\right)
\end{array} \\
& \begin{array}{c|c}
\widetilde{x}=t_{2} & \widetilde{x}=t_{2 i+1}(i=1, \ldots, n-2) \\
\hline \rho_{\boldsymbol{\lambda}}^{\prime}\left(\gamma_{t_{2}}\right)=\left(\begin{array}{cc}
0 & a_{0}^{2} \\
-a_{0}^{-2} & 0
\end{array}\right) & \rho_{\boldsymbol{\lambda}}^{\prime}\left(\gamma_{t_{2 i+1}}\right)=\left(\begin{array}{cc}
a_{i+1} & 0 \\
0 & a_{i+1}^{-1}
\end{array}\right)
\end{array} \\
& \begin{array}{c|c}
\widetilde{x}=t_{2 i+2}(i=1, \ldots, n-2) & \tilde{x}=\infty \\
\hline \rho_{\boldsymbol{\lambda}}^{\prime}\left(\gamma_{t_{2 i+2}}\right)=\left(\begin{array}{cc}
a_{i+1}^{-1} & 0 \\
0 & a_{i+1}
\end{array}\right) & \rho_{\boldsymbol{\lambda}}^{\prime}\left(\gamma_{\infty}\right)=\left(\begin{array}{cc}
a_{0} a_{1}^{-1} & 0 \\
0 & a_{0}^{-1} a_{1}
\end{array}\right)
\end{array}
\end{aligned}
$$

Let $\left\{\nu_{1}, \ldots, \nu_{2 n-2}\right\}$ be the roots of the following equation of degree $2 n-2$ :

$$
\sum_{k=1}^{2 n} \frac{\left(\widetilde{H}_{k}\right)_{12}}{\widetilde{x}-t_{k}}=0 .
$$

For each $\nu_{i}$, we define $\rho_{i}$ by

$$
\rho_{i}:=\sum_{k=1}^{2 n} \frac{\left(\widetilde{H}_{k}\right)_{11}+\frac{\theta_{k}}{2}}{\nu_{i}-t_{k}} .
$$

If a tuple $\left(\widetilde{H}_{i}(t)\right)_{i=1, \ldots, 2 n}$ is a solution of the Schlesinger equations, then the corresponding functions $\nu_{j}\left(t_{1}, \ldots, t_{2 n-2}\right)$ and $\rho_{j}\left(t_{1}, \ldots, t_{2 n-2}\right)(j=1, \ldots$, $2 n-2)$ satisfy the Garnier system $\mathcal{G}_{2 n-2}$ (see [10, Theorem 2.1]).

\subsection{Algebraic solution}

By the morphism (4.2), we have a generically finite morphism $\operatorname{Spec} \mathbb{C}\left[\rho_{i}, \nu_{i}\right]_{1 \leqslant i \leqslant 2 n-2} \times \widetilde{T} \longrightarrow \operatorname{Spec} \mathbb{C}\left[\rho_{i}, \nu_{i}\right]_{1 \leqslant i \leqslant 2 n-2} \times \operatorname{Spec} \mathbb{C}\left[t_{1}, \ldots, t_{2 n-2}\right]$. We consider the algebraic solution of $\mathcal{G}_{2 n-2}$ associated to the representation $\rho_{\boldsymbol{\lambda}}^{\prime}$.

Proof of Theorem 1.4. - For the residue matrices $H_{i}^{\tilde{T}}$ of $\left(\nabla_{\mathbb{P}^{1} \times \tilde{T} / \tilde{T}}\right)_{\boldsymbol{\lambda}}$, we put

$$
\widetilde{H}_{i}^{\tilde{T}}:=\left(\begin{array}{cc}
-1 & a-2 \\
1 & a
\end{array}\right)^{-1} H_{i}^{\tilde{T}}\left(\begin{array}{cc}
-1 & a-2 \\
1 & a
\end{array}\right)
$$


for $i=1, \ldots, 2 n$. Let $\mathcal{A}^{\tilde{T}}(\widetilde{x})$ be the family of the Fuchsian systems with $2 n+1$ regular singularities at $0,1, t_{1}, \ldots, t_{2 n-2}, \infty$ parametrized by $\widetilde{T}$ defined by

$$
\mathcal{A}^{\tilde{T}}(\widetilde{x}):=\mathrm{d}+\widetilde{H}_{2 n-1}^{\tilde{T}} \frac{\mathrm{d} \widetilde{x}}{\widetilde{x}}+\widetilde{H}_{2 n}^{\tilde{T}} \frac{\mathrm{d} \widetilde{x}}{\widetilde{x}-1}+\sum_{i=1}^{2 n-2} \widetilde{H}_{i}^{\tilde{T}} \frac{\mathrm{d} \widetilde{x}}{\widetilde{x}-t_{i}} .
$$

Since $\widetilde{H}_{2 n+1}^{\tilde{T}}:=-\sum_{i=1}^{2 n} \widetilde{H}_{i}^{\tilde{T}}$ is a diagonal matrix, we have the assertion (i) of Theorem 1.4.

By Proposition 3.3, for each $\widetilde{t} \in \widetilde{T}$, the Fuchsian system $\mathcal{A}^{\tilde{T}}(\widetilde{x})$ has the monodromy representation which is conjugated to $\rho_{\boldsymbol{\lambda}}^{\prime}$, which is independent of $\widetilde{t} \in \widetilde{T}$. That is, the family $\mathcal{A}^{\tilde{T}}(\widetilde{x})$ of the Fuchsian systems parametrized by $\widetilde{T}$ preserves their monodromy representations. Then we have the assertion (ii) of Theorem 1.4.

By (4.3), (4.4), and (4.5), we have algebraic functions $\nu_{i}, \rho_{i}(i=1, \ldots$, $2 n-2)$ on $\widetilde{T}$. These algebraic functions give the solution of $\mathcal{G}_{2 n-2}$ associated to the representation $\rho_{\boldsymbol{\lambda}}^{\prime}$. Since $\operatorname{dim} \widetilde{T}=2 n-2$ and $\widetilde{T} \rightarrow \operatorname{Spec} \mathbb{C}\left[t_{1}, t_{2}, \ldots\right.$, $\left.t_{2 n-2}\right]$ is a generically finite morphism, we have the assertion (iii) of Theorem 1.4 .

\section{Acknowledgments}

The author thanks Frank Loray for many valuable discussions. He also thanks Masa-Hiko Saito for warm encouragement. He is grateful to the anonymous referee's suggestions which helped to improve the paper.

\section{Bibliography}

[1] K. Corlette \& C. Simpson, "On the classification of rank-two representations of quasiprojective fundamental groups", Compos. Math. 144 (2008), no. 5, p. 1271-1331.

[2] G. Cousin, "Algebraic isomonodromic deformations of logarithmic connections on the Riemann sphere and finite braid group orbits on character varieties", Math. Ann. 367 (2017), no. 3-4, p. 965-1005.

[3] A. I. Degtyarev, "Quintics in $\mathbb{C P}^{2}$ with nonabelian fundamental group", St. Petersbg. Math. J. 11 (2000), no. 5, p. 809-826.

[4] P. Deligne, Équations différentielles à points singuliers réguliers, Lecture Notes in Mathematics, vol. 163, Springer, 1970.

[5] R. Garnier, "Sur les équations différentielles du troisième ordre dont l'intégrale générale est uniforme et sur une classe d'équations nouvelles d'ordre supérieur dont l'intégrale générale a ses points critiques fixes", Ann. Sci. Éc. Norm. Supér. 29 (1912), p. $1-126$.

[6] _ , "Solution du problème de Riemann pour les systèmes différentiels linéaires du second ordre", Ann. Sci. Éc. Norm. Supér. 43 (1926), p. 239-352. 
A family of flat connections and algebraic Garnier solutions

[7] A. Girand, "A new two-parameter family of isomonodromic deformations over the five punctured sphere", Bull. Soc. Math. Fr. 144 (2016), no. 2, p. 339-368.

[8] H. A. Hamm \& L. D. Tráng, "Un théorème de Zariski du type de Lefschetz", Ann. Sci. Éc. Norm. Supér. 6 (1973), no. 4, p. 317-355.

[9] F. Loray, J. V. Pereira \& F. Touzet, "Representations of quasiprojective groups, Flat connections and Transversely projective foliations", J. Éc. Polytech., Math. 3 (2016), p. 263-308.

[10] M. Mazzocco, "The geometry of the classical solutions of the Garnier systems", Int. Math. Res. Not. 2002 (2002), no. 12, p. 613-646.

[11] К. Окамото, "Isomonodromic deformation and Painlevé equations, and the Garnier system", J. Fac. Sci., Univ. Tokyo, Sect. I A 33 (1986), no. 3, p. 575-618. 